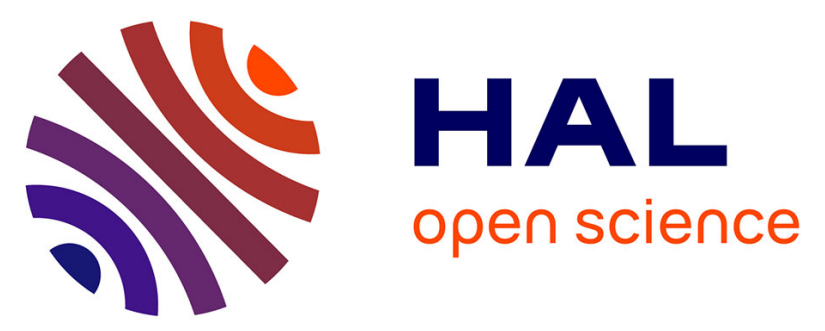

\title{
Atomic force microscopy (AFM), transmission electron microscopy (TEM), and scanning electron microscopy (SEM) of nano-scale plate-shaped second phase particles
}

Ihor Sobchenko, Josef Pesicka, Dietmar Baither, Werner Stracke, Thomas

Pretorius, Lifeng Chi, Rudolf Reichelt, Eckhard Nembach

\section{To cite this version:}

Ihor Sobchenko, Josef Pesicka, Dietmar Baither, Werner Stracke, Thomas Pretorius, et al.. Atomic force microscopy (AFM), transmission electron microscopy (TEM), and scanning electron microscopy (SEM) of nano-scale plate-shaped second phase particles. Philosophical Magazine, 2008, 87 (17), pp.2427-2460. 10.1080/14786430701203184 . hal-00513818

\author{
HAL Id: hal-00513818 \\ https://hal.science/hal-00513818
}

Submitted on 1 Sep 2010

HAL is a multi-disciplinary open access archive for the deposit and dissemination of scientific research documents, whether they are published or not. The documents may come from teaching and research institutions in France or abroad, or from public or private research centers.
L'archive ouverte pluridisciplinaire HAL, est destinée au dépôt et à la diffusion de documents scientifiques de niveau recherche, publiés ou non, émanant des établissements d'enseignement et de recherche français ou étrangers, des laboratoires publics ou privés. 


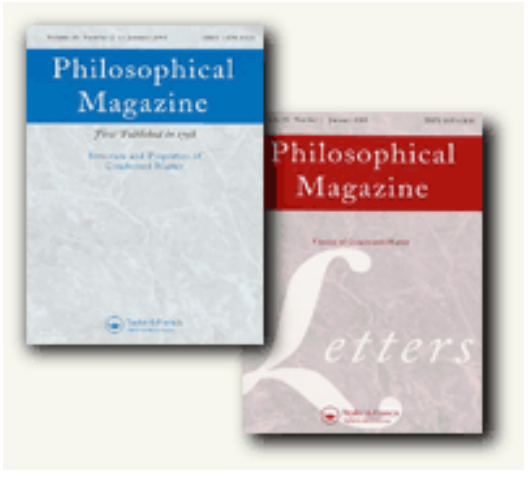

\section{Atomic force microscopy (AFM), transmission electron microscopy (TEM), and scanning electron microscopy (SEM) of nano-scale plate-shaped second phase particles}

\begin{tabular}{|r|l|}
\hline Journal: & Philosophical Magazine \& Philosophical Magazine Letters \\
\hline Manuscript ID: & TPHM-06-Sep-0365.R2 \\
\hline Journal Selection: & Philosophical Magazine \\
\hline Dathor: & 04-Jan-2007 \\
\hline Complete List of Authors: & $\begin{array}{l}\text { Sobchenko, Ihor; University of Muenster, Materialphysik; Cherkasy, } \\
\text { Theoretical Physics } \\
\text { Pesicka, Josef; Charles University, Faculty of Mathematics \& Physics } \\
\text { Baither, Dietmar; University of Muenster, Materialphysik } \\
\text { Stracke, Werne;; Universitaet Muenster, Institut fuer Medizinische } \\
\text { Physik und Biophysik } \\
\text { Pretorius, Thomas; Universitaet Muenster, Materialphysik; Bremer } \\
\text { Institut fuer angewandte Strahltechnik } \\
\text { Chi, Lifeng; Universitaet Muenster, Physikalishes Institut } \\
\text { Reichelt, Rudolf; Universitaet Muenster, Institut fuer Medizinische } \\
\text { Physik und Biophysik } \\
\text { Nembach, Eckhard; University of Muenster, Materialphysik }\end{array}$ \\
\hline Keywords: & $\begin{array}{l}\text { atomic force microscopy, nanoparticles, nanoscale precipitates, Ni- } \\
\text { based superalloys, nickel alloys, phase decomposition, SEM, } \\
\text { transmission electron microscopy }\end{array}$ \\
\hline Keywords (user supplied): & $\begin{array}{l}\text { magnetic force microscopy, superellipsoid, plate-shaped } \\
\text { precipitates }\end{array}$ \\
\hline \hline
\end{tabular}




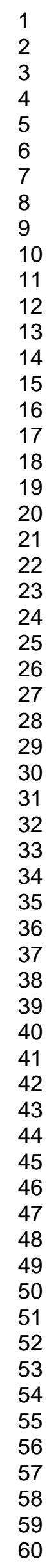

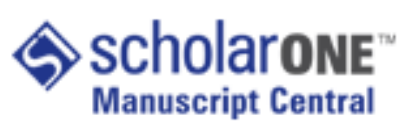

http://mc.manuscriptcentral.com/pm-pml 


\author{
Atomic force microscopy (AFM), transmission electron microscopy (TEM), \\ and scanning electron microscopy (SEM) \\ of nano-scale plate-shaped second phase particles \\ IHOR SOBCHENKO $\uparrow \S$, JOSEF PESICKA $\uparrow+$, DIETMAR BAITHER $\dagger$, \\ WERNER STRACKE\#, THOMAS PRETORIUS $\dagger \llbracket$ II, LIFENG CHIX, \\ RUDOLF REICHELT $\#$, and ECKHARD NEMBACH $\dagger^{*}$ \\ $\dagger$ Institut für Materialphysik, Universität Münster, \\ Wilhelm-Klemm-Strasse 10, 48149 Münster, Germany \\ \# Institut für Medizinische Physik und Biophysik, Universitätsklinikum, Universität Münster, \\ Robert-Koch-Strasse 31, 48149 Münster, Germany \\ × Physikalisches Institut, Universität Münster, \\ Wilhelm-Klemm-Strasse 10, 48149 Münster, Germany \\ $\S$ Permanent address: Department of Theoretical Physics, Cherkasy National University, \\ Shevchenko Blv. 81, 18031 Cherkasy, Ukraine \\ + Permanent address: Charles University, Faculty of Mathematics and Physics, \\ Department of Metal Physics, Ke Karlovu 5, 12116 Prague, Czech Republic \\ II Now with: Bremer Institut für angewandte Strahltechnik, \\ Klagenfurter Strasse 2, 28359 Bremen, Germany \\ * Corresponding author. E-mail: nembach@nwz.uni-muenster.de \\ Phone: ++49-251-83-33570, Fax: ++49-251-83-38346
}

\begin{abstract}
The feasibility of accurately measuring the size and the volume fraction of nano-scale plate-shaped precipitates by atomic force microscopy (AFM) has been explored. For quantitative evaluations their unhandy geometry is conveniently described as superellipsoids. The experimental alloy $\mathrm{Ni}_{69} \mathrm{Co}_{9} \mathrm{Al}_{18} \mathrm{Ti}_{4}$ served as a model system: plate-shaped disordered $\gamma$-precipitates form in the $\mathrm{L1}_{2}$-long-range ordered $\gamma$ '-matrix. The results obtained by AFM are compared with those derived from transmission (TEM) and from high resolution scanning electron microscopy (SEM). The agreement between the AFM and the TEM results is good. In spite of the low number of SEM images taken, the same holds for
\end{abstract}


the SEM results. In addition, magnetic force microscopy was applied; its results are acceptable. The main advantages of AFM are (i) the numerical output for all three dimensions, (ii) the simplicity of its operation, and (iii) the lower cost of the microscope itself. The first point allows to directly subject the numerical AFM output data to automated computer based evaluations. All present experimental and evaluation procedures are also applicable to cube-shaped particles with rounded edges and corners as found e.g. in $\gamma^{\prime}$-strengthened nickel based superalloys.

Keywords: Atomic force microscopy (AFM); Magnetic force microscopy (MFM); Transmission electron microscopy (TEM); Field emission scanning electron microscopy (FESEM); Plate-shaped precipitates; Second phase particles; Nickel based alloy; Nano-structure; Superellipsoid.

\section{Introduction}

Many structural materials derive their high strength from coherent precipitates of secondary phases. Well known examples are Guinier-Preston zones in aluminium based alloys and $\gamma$ '-precipitates in nickel based superalloys [1-3]. The strengthening effects of coherent precipitates are governed by their shape, size, and volume fraction and by intrinsic properties of the two phases involved, e.g. by their crystal structure, their lattice mismatch, and their elastic stiffness. The shape of the precipitates in turn depends on their specific interface energy, their lattice mismatch, and the elastic stiffness of both phases. Anisotropies of these parameters are of great importance. The most common shapes are spheres, cubes, and plates. Plates are favoured by a low specific interface energy, a high lattice mismatch, a low elastic stiffness of the precipitates, and a high precipitate volume [4-6]. In the case of spheres and cubes their size is described by the average radius $r$ and by the average cube length $d$, respectively. To characterise plate-shaped precipitates at least two parameters are required: some linear measure $2 a$ of their average areal extension and their average thickness $2 b$. In addition, the distribution functions $\Phi$ of the individual particle radius $\rho_{\text {i }}$, the cube length $\delta_{i}$, the areal extension $2 \alpha_{i}$, and the thickness $2 \beta_{i}$ are considered.

The size of precipitates relevant for strengthening is in the nanometer range; hence the standard method for the determination of $r, d, a$, and $b$ is transmission electron microscopy (TEM). Since atomic force microscopes (AFM) are much easier to operate and of much lower 
cost, more recently they too have been used to determine the size and the volume fraction of spherical and cubic particles [7-13]. Moreover in contrast to the TEM, also compact specimens can be investigated in the AFM, which facilitates the preparation of the specimens, and thanks to the direct three-dimensional digital output automated computer based evaluation procedures can readily be applied.

In two former investigations [11,12], the present groups successfully used AFMs for the accurate determination of $r, \Phi\left(\rho_{\mathrm{i}}\right)$, and the volume fraction of two different types of spherical nano-scale second phase particles. Elaborate quantification procedures were developed. Special attention had been paid to effects of the finite size of the AFM tip and to the preferential attack of the matrix by the electrolyte. Presently the feasibility to characterise plate-shaped precipitates by AFM has been explored. Their lower symmetry renders their characterisation more involved because (i) the two lengths $a$ and $b$ instead of just one have to be measured and (ii) the often high aspect ratio $a / b$ complicates the choice of the magnification. Good statistics necessitate the evaluation of a high number of particles. Hence the relatively high value of $a$ calls for a low magnification, whereas the smaller thickness $b$ calls for a high magnification. To verify the AFM results they are compared with results derived from TEM and high resolution field emission scanning electron microscopy (FESEM). Since the investigated material is ferromagnetic, also magnetic force microscopy (MFM) was performed. For this purpose the standard AFM tips were replaced by hard magnetic cobalt alloy coated ones. All experimental and evaluation procedures reported below are also applicable to cube-shaped particles with rounded edges and corners; in this case $a$ equals $b$.

The experimental alloy $\mathrm{Ni}_{69} \mathrm{Co}_{9} \mathrm{Al}_{18} \mathrm{Ti}_{4}$ served as a model system. After ageing below about $1380 \mathrm{~K}$, this alloy is two-phase: nano-scale coherent precipitates of the disordered or short-range ordered f.c.c. $\gamma$-phase are embedded in the $\mathrm{L1}_{2}$-long-range ordered $\gamma$ '-phase [1416]. Evidently this arrangement of phases is inverse to that of nickel based superalloys, in which the $\gamma$-phase forms the matrix and particles of the $\gamma$ '-phase precipitate [1-3]. Strengthening of $\mathrm{Ni}_{69} \mathrm{Co}_{9} \mathrm{Al}_{18} \mathrm{Ti}_{4}$ by $\gamma$-precipitates and the relevant dislocation processes were the subjects of two former studies $[15,16]$. In fact, this alloy had been designed as a model system for the investigation of particle strengthening of intermetallic phases. Since the former specimens had been aged for up to $264 \mathrm{~h}$ at $973 \mathrm{~K}$ the $\gamma$-precipitates in them were very small and of approximately spherical shape: $r$ was below $7 \mathrm{~nm}$. The present specimens were aged for 
$333 \mathrm{~h}$ at $1173 \mathrm{~K}$, which resulted in larger and hence plate-shaped $\gamma$-precipitates. TEM, FESEM, AFM, and MFM images are presented in section 4. The present $\gamma^{\prime}$-phase may be considered as a derivative of the well-known intermetallic phase $\mathrm{Ni}_{3} \mathrm{Al}$ [17]. The $\gamma$-precipitates are richer in $\mathrm{Co}$ and poorer in all other constituents than the $\gamma^{\prime}$-matrix. This will be detailed in section 3 .

After the nucleation process of $\gamma$-precipitates is accomplished, the growth of their average size is mainly governed (i) by further depletion of the $\gamma^{\prime}$-matrix of Co and (ii) by dissolution of smaller $\gamma$-precipitates and incorporation of their material by bigger ones. The latter process is analogous to Ostwald ripening of spherical precipitates [18]: the total amount of interface energy is reduced. Pretorius et al. [15] aged $\mathrm{Ni}_{69} \mathrm{Co}_{9} \mathrm{Al}_{18} \mathrm{Ti}_{4}$ isothermally at $973 \mathrm{~K}$ and found that for $r<7 \mathrm{~nm}$ the $\gamma$-volume fraction increased with ageing time.

\section{Crystallography and geometry of the $\gamma$-precipitates}

The crystallography of coherent plate-shaped precipitates determines the optimum imaging geometry. The habit planes of the plate-shaped $\gamma$-precipitates in $\mathrm{Ni}_{69} \mathrm{Co}_{9} \mathrm{Al}_{18} \mathrm{Ti}_{4}$ are of the type $\{001\}$. Therefore the Miller indices of the plane of the thin TEM-foils were chosen to be (001) and the incident electron beam in the TEM and FESEM was normal to them. Other imaging geometries would have complicated the evaluations further. The two families of $\gamma$-plates with the indices (100) and (010) are imaged edge-on, whereas the (001)-family is viewed in-plane. The same foils were studied by the TEM, FESEM, and AFM. The morphology of the $\gamma$-precipitates is in accordance with the observation that Young's modulus of $\gamma^{\prime}$-strengthened superalloys is lowest along <001>-directions [19,20].

'[insert figure 1 about here]'

The $\gamma$-precipitates are neither flat circular cylinders, nor flat cuboids, nor flat standard ellipsoids of revolution (see section 4), but they can be very well described as superellipsoids [21-26]. The coordinates of points on the $\gamma^{\prime}-\gamma$-interface of an (001)-in-plane $\gamma$-precipitate which is centred at the origin of the coordinate system, are

$$
\left(\frac{|x|}{\alpha}\right)^{n}+\left(\frac{|y|}{\alpha}\right)^{n}+\left(\frac{|z|}{\beta}\right)^{k}=1 .
$$


$\alpha$ and $\beta$ are the half-axes with $\alpha>\beta$. The exponents $\_n$ and $k$ are integers exceeding 2 . Evidently the mathematical description of a superellipsoid involves four independent parameters. $\alpha$ appears in the denominator of $|x|$ as well as of $|y|$ because the extension of in-plane (001)- $\gamma$-plates is approximately the same in these two directions (see section 4). Deviations from this symmetry will be discussed below and in section 4.1.2. For $n=k=2$ a standard rotational ellipsoid is obtained and for $n \rightarrow \infty$ and $k \rightarrow \infty$ a cuboid. The effects of $n$ and $k$ on the shape of the superellipsoid are demonstrated in figure 1 for $\alpha=5 \beta$. The intersections of the superellipsoid with the plane characterised by $y \equiv 0$, i.e. edge-on view, and with the plane $z \equiv 0$, i.e. in-plane view, are shown in figures $1(\mathrm{a})$ and (b), respectively. The quoted intersections are superellipses [25,26]. Figure 1(c) gives three-dimensional sketches for four combinations of $n$ and $k$ and thus visualises the effects of these exponents on the shape of the superellipsoid. For the volume $V$ of the superellipsoid holds

$$
V=q_{\mathrm{v}}(n, k) \pi \alpha^{2} \beta
$$

The subscript 'v' of the coefficient $q_{\mathrm{V}}(n, k)$ indicates 'volume'. Some values of $q_{\mathrm{V}}(n, k)$ are listed in figure 1(a). Equation (2b) is an approximate numerical representation of the function $q_{\mathrm{v}}(n, k)$ :

$$
q_{\mathrm{V}}(n, k)=a_{0}+a_{\mathrm{n}} n+a_{\mathrm{nn}} n^{2}+a_{\mathrm{k}} k+a_{\mathrm{kk}} k^{2}+a_{\mathrm{nk}} n k
$$

with $a_{0}=0.1404, a_{\mathrm{n}}=0.4992, a_{\mathrm{nn}}=-0.0383, a_{\mathrm{k}}=0.2369, a_{\mathrm{kk}}=-0.0158, a_{\mathrm{nk}}=-0.0094$. For $n$ and $k$ between 2 and 6 , this approximation is accurate within $\pm 2 \%$ [25].

Deleted: 4

A generalisation of equation (1) is possible by allowing (i) for different lengths of the two longer half-axes and / or (ii) for different exponents, i.e. the first two terms in equation (1) are replaced by $\left(|x| / \alpha_{1}\right)^{n_{1}}$ and $\left(|y| / \alpha_{2}\right)^{n_{2}}$, respectively, with $\alpha_{1} \neq \alpha_{2}$ and / or $n_{1} \neq n_{2}$ [25]. In this case also equation (2a) has to be modified

$$
V=q_{\mathrm{V}}\left(n_{1}, n_{2}, k\right) \pi \alpha_{1} \alpha_{2} \beta .
$$


For later reference the average length $\sigma_{\alpha}(n)$ of chords through the superellipsoid are defined. The average is taken over chords which (i) lie in the (001)-plane (in-plane view) characterised by $z \equiv 0$ and (ii) are parallel to [010], i.e. $\sigma_{\alpha}(n)$ equals the average vertical extension of the sketches in figure 1(b):

$$
\sigma_{\alpha}(n)=q_{\mathrm{C}}(n, n) \quad \alpha \quad \pi \quad .
$$

The subscript 'c' indicates 'chord'. $q_{\mathrm{C}}(n, n)$ equals: $0.5000,0.5623,0.5902,0.6049$,

$2 / \pi=0.6366$ for $n=2,3,4,5, \infty$, respectively [25]. $n=2$ and $\infty$ refer to a circle and a square, respectively. Two more analogous average chords are defined: $\sigma_{\beta}(n, k)$, which refers to chords which (i) lie in the (010)-plane (edge-on view) characterised by $y \equiv 0$ and (ii) are parallel to [001], i.e. to horizontal chords in figure $1(\mathrm{a})$, and $\sigma_{\alpha}(n, k)$, which refers to chords which (i) lie in the (010)-plane (edge-on view) characterised by $y \equiv 0$ and (ii) are parallel to [100], i.e. to vertical chords in figure 1(a).

$$
\begin{array}{llll}
\sigma_{\beta}(n, k)=q_{\mathrm{C}}(n, k) & \beta & \pi \\
\sigma_{\alpha}(n, k) & =q_{\mathrm{C}}(n, k) & \alpha & \pi
\end{array}
$$

$q_{\mathrm{C}}(n, k)$ is symmetric, i.e. $q_{\mathrm{C}}(n, k)=q_{\mathrm{C}}(k, n)$. Equation (3d) is a numerical approximation of the function $q_{\mathrm{C}}(n, k)$; for $n$ and $k$ between 2 and 6 , it is accurate within $\pm 1.5 \%$ :

$$
q_{\mathrm{C}}(n, k)=a_{0}+a_{\mathrm{n}}(n+k)+a_{\mathrm{nn}}\left(n^{2}+k^{2}\right)+a_{\mathrm{nk}} n k
$$

with $a_{0}=0.3573, a_{\mathrm{n}}=0.0458, a_{\mathrm{nn}}=-0.0028, a_{\mathrm{nk}}=-0.0027$.

With the aid of equations (3b) and (3c) the area $\mathbf{A}(\alpha, \beta, n, k)$ of a superellipse which is characterised by the parameters $\alpha, \beta, n$, and $k$, can be calculated

$$
\mathbf{A}(\alpha, \beta, n, k)=2 \alpha q_{\mathrm{c}}(n, k) \beta \pi
$$

Applying equations (1) - (3), all relevant geometric parameters of any type of convex particles, e.g. of cubes with rounded edges and corners, can easily be calculated: volume, area 
of the particle / matrix interface, intersections of the particle with any crystallographic plane, chords. The $\gamma$ '-precipitates in the most advanced nickel based superalloys are such cubes [3,79].

\section{Experiments}

Slices of $0.3 \mathrm{~mm}$ thickness were spark cut from aged $\mathrm{Ni}_{69} \mathrm{Co}_{9} \mathrm{Al}_{18} \mathrm{Ti}_{4}$-single crystals, ground, and twin-jet electro-polished (electro-polishing machine: Tenupol-3 [Struers, Copenhagen, Denmark]; electrolyte: $20 \mathrm{ml}$ perchloric acid (63\%) in 80ml ethanol; temperature: $240 \mathrm{~K}$; voltage: $25 \mathrm{~V}$ ). With the following two exceptions the preparation of the thin TEM-foils was the same as in Refs. [15,16]: (i) the present ageing conditions were $333 \mathrm{~h}$ at $1173 \mathrm{~K}$, which resulted in plate-shaped, instead of spherical (see section 1) $\gamma$-precipitates, and (ii) the shape and crystallography of the $\gamma$-precipitates required that the indices of the plane of the foils were (001) (see section 2). The present ageing treatment was preceded by a $\gamma$-nucleation treatment: $24 \mathrm{~h}$ at $978 \mathrm{~K}$. As described by Baither et al. [16], the aged material contained some widely spaced irregular arrangements of the two phases. This was due to dendritic growth of the single crystals. Since the spacing of these irregularities was around $0.3 \mathrm{~mm}$, they could easily be avoided in the microscopes.

Without any additional preparation specific to the applied microscope, the same foils were studied by TEM, FESEM, and AFM. All micrographs were taken at ambient temperature. Two AFMs, AFM1 and AFM2, were used, both with two different types of tips, Tip1 and Tip2. The TEM, the FESEM, and AFM1 were the same ones as in the previous studies of spherical particles [12,16]. AFM1: AP-100 Autoprobe CP (Park Scientific Instruments, Sunnyvale, USA); AFM2: Dimension ${ }^{\mathrm{TM}} 3000$ (Digital Instruments, Santa Barbara, USA); 200kV-TEM: 800NA (Hitachi, Tokyo, Japan); FESEM: S-5000 (Hitachi, Tokyo, Japan). Tip1: SuperSharpSilicon ${ }^{\mathrm{TM}}$ (tip radius $<5 \mathrm{~nm}$, typical $\approx 2 \mathrm{~nm}$; half-cone angle of the outermost $200 \mathrm{~nm}$ of the tip $<10^{\circ}$ ); Tip2: PointProbe ${ }^{\oplus}$ (tip radius $<10 \mathrm{~nm}$, typical $<7 \mathrm{~nm}$; half-cone angle $\approx 20^{\circ}$, but $<10^{\circ}$ at the very end of the tip). Both types of tips are produced by NANOSENSORS ${ }^{\mathrm{TM}}$, Neuchâtel, Switzerland, who also provided the quoted specifications. The tip radii and the cone angles were checked by FESEM and TEM. TEM yielded the result that for Tip2 the radius is indeed below 10nm and the half-one angle - measured over the outermost $150 \mathrm{~nm}-$ does not exceed $15^{\circ}$. Since Tip 1 is very fragile, its first approach to the specimen required utmost care. Both AFMs are constructed such that the tip of the monolithic 
cantilevers is inclined by $12^{\circ}$ in AFM1 and by $10^{\circ}$ in AFM2, away from the normal. Scans were performed along two alternative orthogonal directions: from side to side and from front to back. The quality of the topographs obtained was the same for both directions. The magnifications of the microscopes were calibrated; hence possible remaining errors are estimated to be at most $0.5 \%$ for the TEM, $2 \%$ for the FESEM, $2 \%$ for AFM 1 , and $5 \%$ for AFM2.

FESEM and AFM revealed that the electrolyte attacks the $\gamma$-precipitates more strongly than the $\gamma^{6}$-matrix (see sections 4.2 and 4.3). Hence they appear as pits in the FESEM and AFM images. In the following the pits left behind by the etched away parts of the $\gamma$-precipitates will be referred to as $\gamma$-pits. The present etching effect is the same one as that observed for nickel based superalloys, in which the $\gamma$-matrix was more strongly attacked than the strengthening $\gamma^{\prime}$-precipitates, which stuck out of the foils [12]. It is emphasised that etching was not intended, but was a mere side effect of the standard electro-polishing procedure of thin TEM-foils. Since the same specimens were to be studied in all three types of microscopes (TEM, FESEM, AFM), the requirements of the TEM had to be fulfilled.

The present material $\mathrm{Ni}_{69} \mathrm{Co}_{9} \mathrm{Al}_{18} \mathrm{Ti}_{4}$ is ferromagnetic at ambient temperature. Hence also magnetic force microscopy (MFM) could be performed: AFM2 was operated with a tip of the type PointProbe-MFMR, which had a 40nm thick cobalt alloy coating. Its producer NANOSENSORS ${ }^{\mathrm{TM}}$ specifies that the typical tip radius is less than $50 \mathrm{~nm}$. The preparation of the specimens investigated by MFM was similar to that of those studied by the other microscopes, but they were only mechanically ground and polished, finally with $250 \mathrm{~nm}$ diamond paste. There was no electro-polish and hence no etching. The root mean square roughness measured by AFM2 equipped with a magnetic tip was less than $2.5 \mathrm{~nm}$.

While the TEM produces a parallel projection of the volume of the thin foil into a plane perpendicular to the optical axis, the AFM and the FESEM yield three-dimensional information on the surface profile of the foil and the MFM registers differences in magnetic field above the surface. In the evaluations, allowances are made for the fact that even $\gamma$-precipitates whose centres lie outside the thin foil, may yield contrast.

The compositions of both phases of the thin foils were determined by energy dispersive X-ray (EDX) analyses in the TEM. The following atomic percentages were found 
in the $\gamma^{\prime}-$ / $\gamma$-phase: Ni 68.6 / 65.1, Co 9.5 / 21.9, Al 17.8 / 12.5, Ti 4.1 / 0.5. The error limits of these concentrations are estimated to be between 1 and 2at.\%. In agreement with common

knowledge [e.g. 2, 27] Co preferentially partitions to the $\gamma$-phase and Al and Ti to the

Deleted: 6 $\gamma^{\prime}$-phase.

\section{Data evaluations}

Since TEM, SEM, AFM, and MFM images require different evaluation procedures, the respective analyses are presented in separate sections. The same holds for edge-on and in-plane $\gamma$-particles. It is stressed that the planes of all micrographs, presented as well as evaluated ones, are parallel to the (001)-plane of the TEM-foil. So far no automated computer based evaluation procedure was implemented, because it would require the exact standardisation of all processes. In routine work, however, such procedures are very helpful.

\subsection{Transmission electron microscopy}

The $\mathrm{L1}_{2}$-long-range ordered $\gamma^{\prime}$-precipitates in superalloys are normally dark-field imaged with one of their superlattice reflections. Due to the inverse arrangement of the $\gamma$ - and $\gamma^{\prime}$-phase in $\mathrm{Ni}_{69} \mathrm{Co}_{9} \mathrm{Al}_{18} \mathrm{Ti}_{4}$ (see section 1), presently bright field images were taken. An example is presented in figure 2 . The standard magnification was $10,000 \mathrm{x}$ and the photographic negatives were scanned with 600dpi. Four to ten scanned neighbouring original micrographs were 'pasted together' in the computer and treated as one micrograph. The evaluation of each pasted together micrograph started with a search for those $\gamma$-pits whose entire images were in the micrograph. Subsequently the area $A$ was delimited by straight lines such that the centres of the 'entire' $\gamma$-images lay within $A$. Evidently some margins which contained partially imaged $\gamma$-pits, were disregarded. Tracing out the evaluated area $A$ involved some arbitrariness, which is allowed for in the quoted error limits. The percentage of the respective error decreases as $A$ increases. Pasting together helped to use the original micrographs more efficiently because fewer margins containing partially imaged $\gamma$-pits had to be disregarded. Analogous procedures were applied to the FESEM, AFM, and MFM images. The thickness $t$ of the thin TEM-foils was determined by convergent beam electron diffraction. Within the experimental limits of error of $10 \%, t$ was constant throughout each pasted together micrograph. Six TEM-foils were studied; they yielded nine pasted together areas taken at different parts of the foils. The same areas were evaluated for edge-on and for in-plane $\gamma$-pits. 
4.1.1 Edge-on $\gamma$-precipitates. Often the strongly bright mass thickness contrast generated by edge-on $\gamma$-pits is surrounded by a region of weaker contrast. It is due to the above mentioned dissolution of the $\gamma$-matrix, especially at the edges of the $\gamma$-pits, and perhaps to some strain. The area of the original, i.e. not pasted together standard micrograph corresponds to about $60(\mu \mathrm{m})^{2}$ of the thin foil. In most cases this area is dominated by either the (100)- or the (010)-family of edge-on $\gamma$-pits. In figure 2, about $60 \%$ of them belong to the (100)-family.

This dominance is probably caused by an elastic interaction between the $\gamma$-precipitates [4,28], which gives rise to a growth selection. The interaction in turn is due to their lattice mismatch.

On the basis of their brightness three types of images of edge-on $\gamma$-pits are distinguished in figure 2 : (i) at $\mathrm{A}$, (ii) at $\mathrm{B}$, and (iii) at $\mathrm{C}$. Tilting through high angles $\left(\approx 35^{\circ}\right)$ and taking micrographs under two beam conditions yielded the information communicated below. 
(i) A: very bright; the edge-on $\gamma$-precipitate led to a deep $\gamma$-pit in at least one surface of the thin foil. A $\gamma$-precipitate which intersects both surfaces gives rise to pits in both of them. In the latter case even holes may appear.

(ii) $\quad \mathrm{B}$ : dark; the edge-on $\gamma$-precipitate lies entirely within the foil and does not contact either surface; there is only strain contrast due to the elastic distortion of the lattice planes. Since the $\gamma$ - and the $\gamma^{\prime}$-phase have nearly the same average atomic number (see section 3) there is hardly any mass thickness contrast.

(iii) C: moderate brightness; the edge-on $\gamma$-precipitate led to a shallow $\gamma$-pit in one surface of the thin foil.

At $\mathrm{A}$ in figure 2, two parallel $\gamma$-pits are very close together; at D the two images are nearly in line. The intersections between the edge-on $\gamma$-precipitate at $E$ with the two surfaces of the foil have different lengths. The longer one is evaluated. At F, two $\gamma$-pit images cross each other. In this case one edge-on $\gamma$-precipitate intersects the upper and one the lower surface of the foil. On the basis of detailed analyses of the strain contrast patterns it is concluded that the two $\gamma$-precipitates do not contact each other. At G, the images of two parallel edge-on $\gamma$-pits superimpose with the image of an in-plane one.

The full axes $2 \alpha_{\mathrm{ij}}^{\prime}$ and $2 \beta_{\mathrm{ij}}^{\prime}$ of the individual $\gamma$-precipitate images are measured by manually marking the $\gamma$-pits. The subscripts $\mathrm{i}$ and $\mathrm{j}$ refer to the number of the evaluated pasted together micrograph $(i \leq Z=9)$ and to the number of the individual $\gamma$-precipitate $\left(1 \leq j \leq N_{\mathrm{i}}\right)$ in micrograph No. i, respectively. Great care is exerted to only allow for the strong central mass thickness contrast and to disregard the weaker one reaching out further. The integer exponents $n$ and $k$ are chosen individually for each $\gamma$-pit such that the superellipse drawn according to equation (1) follows the TEM image well. In most (96\%) cases, $n=k=3$ yield a very good representation. The overall averages over $n$ and $k$ are 2.99 and 3.03, respectively. A total of 4889 edge-on $\gamma$-pits is evaluated, on an average about 100 per original micrograph (area: $\approx 60(\mu \mathrm{m})^{2}$ ). As detailed below, the described procedures do not yield the true half-axes $\alpha_{\mathrm{ij}}$ and $\beta_{\mathrm{ij}-}$ of the $\gamma$-precipitates, but the apparent ones $\alpha_{\mathrm{ij}}^{\prime}$ and $\beta_{\mathrm{ij}}^{\prime}$.

The $\gamma$-precipitates were partially or entirely etched away (see section 3 ). Not only those of them produced contrast in the TEM whose centres lay within the thin foil (in-foil 
$\gamma$-precipitates), but also those whose centres lay outside the foil (out-of-foil $\gamma$-precipitates), however, not more than $\left(\alpha_{\mathrm{ij}}-w\right)$ away from a surface of the foil. If and only if the depth of the $\gamma$-pit marking the position of the $\gamma$-precipitate exceeds the minimum depth $w$, sufficient contrast is obtained. At F, figure 2 shows the superimposed images of a (100)- and of a (010)-edge-on $\gamma$-pit. The contrast of both of them is strong. As communicated above, there is no contact between the two $\gamma$-precipitates. At least the depth of one $\gamma$-pit cannot exceed half of the thickness $t$ of the foil. In figure 2, $t$ is $352 \mathrm{~nm}$. Rather shallow edge-on $\gamma$-pits were imaged under standard [001]-conditions and after tilting through about $35^{\circ}$. Comparisons of theses images led to the conclusion that $w$ is around $0.1^{\bullet} t$. Hence in all evaluations $0.1^{\cdot} t$ is inserted for $w$. The effects of inserting alternative values for $w$ will be discussed towards the end of this section.

In the evaluations, it is assumed (i) that in-foil $\gamma$-precipitates are imaged with their true half-axes $\alpha_{\mathrm{ij}}$ and $\beta_{\mathrm{ij}}$ and (ii) that the $\gamma$-pit images of out-of-foil $\gamma$-precipitates yield chords (see equations (3)). Hence in the case of $w=0$, on an average $\alpha_{\mathrm{ij}}^{\prime}$ and $\beta_{\mathrm{ij}}^{\prime}$ of out-of-foil $\gamma$-precipitates equal $1 / 2 \sigma_{\alpha}(n)$ and $1 / 2 \sigma_{\beta}(n, k)$, respectively. With the exception of $\gamma$-images of type (ii) (see at B in figure 2) one does not know whether a given $\gamma$-image represents an in-foil or an out-of-foil $\gamma$-precipitate. Therefore corrections are performed on the basis of equations (4), which represent weighted averages over both types of $\gamma$-precipitates. The weights are $t$ and $\left[2\left(\alpha_{\mathrm{ij}}-w\right)\right]$ for in-foil and out-of-foil $\gamma$-precipitates, respectively. The factor 2 allows for the two surfaces of the foil.

$$
\begin{aligned}
& \alpha_{\mathrm{ij}}^{\prime}=\frac{t \alpha_{\mathrm{ij}}+\left(\alpha_{\mathrm{ij}}-w\right) \sigma_{\alpha}(n)}{t+2\left(\alpha_{\mathrm{ij}}-w\right)} \\
& \beta_{\mathrm{ij}}^{\prime}=\frac{t \beta_{\mathrm{ij}}+\left(\alpha_{\mathrm{ij}}-w\right) \sigma_{\beta}(n, k)}{t+2\left(\alpha_{\mathrm{ij}}-w\right)}
\end{aligned}
$$

If $n$ equals $k$, the ratios $\alpha_{\mathrm{ij}}^{\prime} / \alpha_{\mathrm{ij}}$ and $\beta_{\mathrm{ij}}^{\prime} / \beta_{\mathrm{ij}}$ are the same. The centres of all visible $\gamma$-precipitates with $\alpha_{\mathrm{ij}} \leq w$ lie within the thin foil. Hence $\alpha_{\mathrm{ij}}=\alpha_{\mathrm{ij}}^{\prime}$ and $\beta_{\mathrm{ij}}=\beta_{\mathrm{ij}}^{\prime}$ hold for them. Inversion of the two above equations yields: 


$$
\begin{gathered}
\alpha_{\mathrm{ij}}=\frac{-\left(t-q_{\mathrm{C}}(n, n) \pi w-2 \alpha_{\mathrm{ij}}^{\prime}\right)+\left|\left[\left(t-q_{\mathrm{C}}(n, n) \pi w-2 \alpha_{\mathrm{ij}}^{\prime}\right)^{2}+4 q_{\mathrm{C}}(n, n) \pi \alpha_{\mathrm{ij}}^{\prime}(t-2 w)\right]^{1 / 2}\right|}{2 q_{\mathrm{C}}(n, n) \pi} \\
\beta_{\mathrm{ij}}=\frac{\beta_{\mathrm{ij}}^{\prime}\left[t+2\left(\alpha_{\mathrm{ij}}-w\right)\right]}{t+\left(\alpha_{i \mathrm{j}}-w\right) q_{\mathrm{C}}(n, k) \pi}
\end{gathered}
$$

On an average, $\alpha_{\mathrm{ij}}$ and $\beta_{\mathrm{ij}}$ are about 7\% larger than $\alpha_{\mathrm{ij}}^{\prime}$ and $\beta_{\mathrm{ij}}^{\prime}$, respectively. $\sigma_{\alpha}(n)$ and $\sigma_{\beta}(n, k)$ represent average chords calculated for $w=0$. Hence it is not quite correct to insert them into equations (4): analogous average chords calculated under disregard of the outermost parts (of extension $w$ ) of the $\gamma$-precipitate should be used instead. Equations (4) overestimate the corrections slightly.

The depth of a $\gamma$-pit left behind by an out-of-foil $\gamma$-precipitate exceeds $w$ if and only if the original position of the $\gamma$-particle centre was not more than $\left(\alpha_{\mathrm{ij}}-w\right), 1 \leq \mathrm{i} \leq \mathrm{Z}=9,1 \leq \mathrm{j} \leq N_{\mathrm{i}}$, away from a $\gamma^{\prime}$-surface. There are $Z=9$ pasted together micrographs and $N_{\mathrm{i}}$ images of edge-on $\gamma$-pits in micrograph No. i. Evidently large $\gamma$-precipitates are favoured. Therefore the weights $Q_{\mathrm{ij}}$, which are proportional to $\left\{1 /\left[t_{\mathrm{i}}+2\left(\alpha_{\mathrm{ij}}-w\right)\right]\right\}$, are used in the calculations of the average half-axes $a_{\mathrm{i}}$ and $b_{\mathrm{i}}$, averaged over micrograph No. i. This weighting procedure is analogous to that in equations (4).

$$
\begin{aligned}
& a_{\mathrm{i}}=\sum_{j=1}^{N_{\mathrm{i}}} \alpha_{\mathrm{ij}} Q_{\mathrm{ij}} \\
& b_{\mathrm{i}}=\sum_{j=1}^{N_{\mathrm{i}}} \beta_{\mathrm{ij}} Q_{\mathrm{ij}}
\end{aligned}
$$

with

$$
Q_{\mathrm{ij}}=\frac{1}{S_{\mathrm{i}}} \frac{1}{t_{\mathrm{i}}+2\left(\alpha_{\mathrm{ij}}-w\right)}
$$

and

$$
S_{\mathrm{i}}=\sum_{\mathrm{m}=1}^{N_{\mathrm{i}}} \frac{1}{t_{\mathrm{i}}+2\left(\alpha_{\mathrm{im}}-w\right)}
$$


Let $A_{\mathrm{i}}$ be the evaluated area of micrograph No. i and $f_{\mathrm{i}}$ the total $\gamma$-volume fraction. 'total' means that the in-plane $\gamma$-precipitates are included; this introduces the factor 1.5. Thus one obtains for $f_{\mathrm{i}}$

$$
\Omega_{\mathrm{ij}}=q_{\mathrm{v}}(n, k) \pi \alpha_{\mathrm{ij}}^{2} \quad \beta_{\mathrm{ij}}
$$

The average volume $V_{\mathrm{i}}$ of edge-on $\gamma$-plates in micrograph No. $\mathrm{i}$ is given by

$$
V_{\mathrm{i}}=\sum_{j=1}^{N_{\mathrm{i}}} \Omega_{\mathrm{ij}} Q_{\mathrm{ij}}
$$

It is emphasised that the summation is carried out only over edge-on $\gamma$-pits. Due to the above mentioned predominance of one family of $\{001\}-\gamma$-precipitates in each original micrograph, the factor 1.5 needs justification. The final result for the $\gamma$-volume fraction $f$ is the average over $Z=9$ pasted together micrographs taken at various areas of six thin foils (see above). Hence any predominance averages out. To conclude the average aspect ratio $R_{\mathrm{i}}$ is defined

$$
R_{\mathrm{i}}=\sum_{j=1}^{N_{\mathrm{i}}} \frac{\alpha_{\mathrm{ij}}}{\beta_{\mathrm{ij}}} Q_{\mathrm{ij}}
$$

The final results for the half-axes $a$ and $b$, the aspect ratio $R$, the volume $V$, and the total $\gamma$-volume fraction $f$ are the weighted averages over $a_{\mathrm{i}}, b_{\mathrm{i}}, R_{\mathrm{i}}, V_{\mathrm{i}}$, and $f_{\mathrm{i}}$, respectively, $1 \leq \mathrm{i} \leq$ $Z=9$. The weights are $\left(N_{\mathrm{i}} / \sum_{\mathrm{m}=1}^{Z} N_{\mathrm{m}}\right)$. The results have been compiled in table 1 . The statistical error limit quoted for $a$ and $b$ is the sum over the standard deviation of the respective average 
and an allowance of $0.5 \%$ for the uncertainty of the magnification of the TEM. In the case of $f$, additional $10 \%$ are added for possible inaccuracies of the foil-thickness and $3 \%$ for uncertainties in tracing out $A_{\mathrm{i}}$. Throughout the following, such sums of errors will be referred to as statistical error limits; they include no allowances for systematic errors, e.g. for misinterpretations of the contrast. The distribution functions $\Phi\left(\alpha_{\mathrm{ij}} / a\right)$ and $\Phi\left(\beta_{\mathrm{ij}} / b\right)$ are shown in figure 3. Again the weights $Q_{\mathrm{ij}}$ are used. The full widths at half maximum are 0.94 and 0.60 , respectively. Both distributions can be very well fitted by a log-normal law. In

Deleted: are perfectly figure 3(c), $\beta_{\mathrm{ij}}$ is plotted versus $\alpha_{\mathrm{ij}}$, no weighting procedure is performed for this latter diagram. Though the scatter of the data is rather wide, the aspect ratio $R_{\mathrm{ij}}=\alpha_{\mathrm{ij}} / \beta_{\mathrm{ij}}$ is clearly $\underline{\text { seen to increase with }} \alpha_{\mathrm{ij}}$ for small and medium sized $\alpha_{\mathrm{ij}}$ and to level off for large $\alpha_{\mathrm{ij} \_}$. Hence it is only of limited value to quote an overall average $R$. Since the strain energy of $\gamma$-precipitates increases approximately as $\beta_{\mathrm{ij}}^{2}, \beta_{\mathrm{ij}}$ of only few of them exceeds 40nm and the distribution function $\Phi\left(\beta_{\mathrm{ij}} / b\right)$ is more narrow than $\Phi\left(\alpha_{\mathrm{ij}} / a\right)$. For $\alpha_{\mathrm{ij}} \leq 700 \mathrm{~nm}$, the function $\beta_{\mathrm{ij}}\left(\alpha_{\mathrm{ij}}\right)$ can be well represented by a polynomial:

$$
\beta_{\mathrm{ij}}=h_{0}+h_{1} \alpha_{\mathrm{ij}}+h_{2} \alpha_{\mathrm{ij}}^{2}
$$

with $h_{0}=12.97 \mathrm{~nm}, h_{1}=0.0772$, and $h_{2}=-0.5463 \cdot 10^{-4}(\mathrm{~nm})^{-1}$.

'[insert table 1 and figure 3 about here]'

The stability of the final results has been checked by trying alternative input values for

Deleted: Since the strain energy of $\gamma$-precipitates increases approximately as $\beta_{\mathrm{ij}}^{2}, \beta_{\mathrm{ij}}$ of only few of them exceeds $40 \mathrm{~nm}$ and the distribution function $\Phi\left(\beta_{\mathrm{ij}} / b\right)$ is more narrow than $\Phi\left(\alpha_{\mathrm{ij}} / a\right)$. $n, k$, and $w$. Setting all exponents $n$ and $k$ equal to 3.0 or varying $w$ between 0.0 and $0.2 \cdot t$ ( $t=$ thickness of the foil) alters the results for $a, b, V$, and $f$ only within their standard deviations.

The present number 4889 of evaluated edge-on $\gamma$-precipitates is probably higher than necessary. In former TEM-studies of spherical nano-scale particles, about 1000 of them were found to be sufficient [2]. Presently, however, there must be enough micrographs taken at different parts of the specimen to average out the above mentioned predominance of one family of $\{001\}-\gamma$-precipitates; otherwise the final result for $f$ is inaccurate. 
4.1.2 In-plane $\gamma$-precipitates. In figure 2 four types of images of them can be distinguished:

(i) at $\mathrm{H}$, (ii) at I, (iii) at J, and (iv) at $\mathrm{K}$.

(i) $\mathrm{H}$ : There is only strain contrast due to the distortion of the lattice planes. This in-plane $\gamma$-precipitate lies entirely deep within the foil and has no contact with either surface. Therefore no etching effects can be discerned. Since the average atomic numbers of the $\gamma$ - and the $\gamma^{\prime}$-phase are nearly the same, hardly any mass thickness contrast is generated.

(ii) I: This situation is analogous to the edge-on one at $\mathrm{D}$, but the two $\gamma$-precipitates have grown together. As detailed below, both long half-axes $\alpha_{\mathrm{ij}}$ are evaluated. This $\gamma$-precipitate has no contact with either surface of the thin foil.

(iii) J: This $\gamma$-precipitate has been completely dissolved; hence no strain, but only mass thickness contrast is visible. There is overlap of this $\gamma$-image with one of type (ii). Etching of the strain field originally lying between the surface of the foil and an in-plane $\gamma$-precipitate may raise the depth of its pit above the actual thickness of the $\gamma$-precipitate.

(iv) K: This $\gamma$-precipitate has been partially etched away. Two $\gamma$-pits appeared; their boundaries are, however, not sharp. The slopes of the upper $\gamma$-pit probably have strong $\{111\}$-components (see sections 4.2 and 4.3). $\{111\}$-planes intersect the surface of the specimen along $<011>$-directions. There is strain contrast due to the elastic distortion of the lattice planes as well as mass thickness contrast. The slight constriction is reminiscent of the edge-on view at $\mathrm{D}$.

Part of the above information was gained from micrographs taken after tilting the foil through high $\left(\approx 35^{\circ}\right)$ angles or under kinematic two beam conditions. These in-plane $\gamma$-pit images clearly show that the $\gamma$-precipitates are not standard rotational ellipsoids.

The average longer half-axis $\alpha_{\mathrm{ij}}$ of in-plane $\gamma$-precipitates can be derived from such micrographs. Only type (i) (H in figure 2) and type (ii) ( I in figure 2) images are evaluated; there are 899 of them. The superellipses are drawn in the middle of the dark contrast (see figure 2, right small micrograph). They directly yield $\alpha_{\mathrm{ij}}$, not only the apparent axis $\alpha_{\mathrm{ij}}^{\prime}$. No 
weighting procedure as in equation (5a) is performed. This is actually not quite correct: since the $\gamma$-precipitates which produce these types of images, do not contact either surface, weights approximately proportional to $\left[1 /\left(t_{\mathrm{i}}-2 \beta_{\mathrm{ij}}\right)\right]$ would be appropriate. However, $\beta_{\mathrm{ij}}$ of in-plane $\gamma$-precipitates is not known. Since $\beta_{\mathrm{ij}}$ increases with $\alpha_{\mathrm{ij}}$ (see figure 3(c)), the disregard of the weights favours small $\gamma$-precipitates and thus leads to a slight underestimate of $1-2 \%$ of the average half-axis $a$. Since the growth of each $\gamma$-precipitate is influenced by its neighbours, its two half-axes $\alpha_{\mathrm{ij}}$ measured along [100] and [010] differ sometimes, e.g. at I in figure 2. Such a case has been mentioned in connection with the introduction of equation (2c). On an average the difference between the two half-axes $\alpha_{\mathrm{ij}}$ of in-plane $\gamma$-precipitates amounts to $\pm 10 \%$. Their arithmetic mean is used. This is analogous to the procedures described in section 4.1.1 for edge-on $\gamma$-precipitates: for them only one long half-axis $\alpha_{\mathrm{ij}}$ was measured and averaged over all evaluated $\gamma$-precipitates. Presently the average geometric mean of the two half-axes $\alpha_{\mathrm{ij}}$ is only $1.1 \%$ smaller than their arithmetic mean. Type (iii) and type (iv) images are not evaluated, because the respective results are very sensitive to the etch and to the choice of the minimum depth $w$. The final result for the half-axis $a_{\text {in-plane }}$ of in-plane $\gamma$-precipitates is the weighted average over all evaluated micrographs. The weighting procedure is analogous to that applied in the case of edge-on $\gamma$-precipitates (see section 4.1.1). The result is listed in table 1 . Evidently $a_{\text {in-plane }}$ agrees within $\pm 2.6 \%$ with its counterpart $a_{\text {edge-on }}$; this agreement is quite gratifying. The $\gamma$-volume fraction cannot be derived from in-plane $\gamma$-precipitates alone, because they yield no information about the shorter half-axis $b$.

The total number of evaluated in-plane $\gamma$-precipitates of types (i) and (ii) is 899 . This number is compared with an estimate derived from edge-on $\gamma$-precipitates:

$$
N_{\text {in-plane }}=\frac{1}{3} \sum_{\mathrm{i}=1}^{Z} f_{\mathrm{i}}\left(t_{\mathrm{i}}-2 b_{\mathrm{i}}\right) A_{\mathrm{i}} / V_{\mathrm{i}}
$$

with $f_{\mathrm{i}}=$ total $\gamma$-volume fraction, $V_{\mathrm{i}}=$ average volume of a $\gamma$-precipitate, $t_{\mathrm{i}}=$ thickness of the foil, $b_{\mathrm{i}}=$ average length of the shorter half-axis, and $A_{\mathrm{i}}=$ evaluated area, $1 \leq i \leq Z=9=$ total number of pasted together micrographs. The averages are taken over $A_{\mathrm{i}}$. This calculation yields $N_{\text {in-plane }}=946$, which agrees within $\pm 2.6 \%$ with the above quoted experimental datum. 
High resolution FESEM images of a thin TEM-foil were recorded with secondary electrons (SE) at $10 \mathrm{kV}$ on photographic film. The tilting angle of the foil was $0^{\circ}$ for the standard micrographs, $\pm 5^{\circ}$ for stereo pairs, and $\pm 30^{\circ}$ for some special purposes. Figure 4 shows two micrographs: the edge-on $\gamma$-pits are typical, the shape of in-plane ones, however, often strongly differs from that in figure 4(b) (see below). The intensity $I$ of the brightness in the positive prints is a monotonically increasing function of the SE intensity $I_{\mathrm{SE}}$. The ratio $I / I_{\mathrm{SE}}$ is affected by the photographic processes applied to the negatives and prints. However, the photographic processes are performed according to a standardised protocol; hence all recorded negative films and the prints made from these films transfer $I_{\mathrm{SE}}$ into the brightness of the prints reproducibly. $I_{\mathrm{SE}}$ strongly depends on [29]: (i) the angle between the incident electron beam and the normal of the SE emitting area, (ii) the inclination of this area relative to the SE-detector, and (iii) the thickness $t$ of the foil; this, however, only if $t$ is smaller than the range of the incident electrons. Moreover, $I_{\mathrm{SE}}$ is strongly enhanced at edges. Three extended regions are distinguished in figures 4 (a) and (b):

Region (1): $I$ is very low in the centres of the $\gamma$-pit images; in this region $I$ of edge-on $\gamma$-precipitates vanishes.

Region (2): I is high.

Region (3): $I$ is moderate.

Region (1) corresponds to the bottom of the $\gamma$-pit, from which - in the case of edge-on $\gamma$-pits zero and - in the case of in-plane ones - less SEs than from the $\gamma^{\prime}$-surface reach the detector. Region (3) is the $\gamma^{\prime}$-surface. These regions are also noticeable in the diagrams $I(x)$ and $I(y)$ shown in figures 4(d), (e), and (g). Based on stereo pairs (see figure 5) and on AFM data (see section 4.3) it is concluded that Regions (2) are plane and parallel to \{011\}-planes of the thin 
foil. Its envisaged surface profile is sketched in figure (6). If the foil had been strongly etched Regions (2) are sometimes parallel to $\{111\}$ (see section 4.3). $\{111\}$-planes intersect the (001)-surface of the specimen along <011>-directions. If etching of an in-plane $\gamma$-precipitate just starts as at $\mathrm{K}$ in figure 2 or if the level of the $\gamma^{\prime}$-matrix reaches the original bottom of the in-plane $\gamma$-precipitate, the resulting $\gamma$-pit deviates from that in figure 4(b): the $\gamma$-pit becomes nearly circular or changes its orientation. This will be discussed further in section 4.3.1. In the

case of edge-on $\gamma$-pits, Region (2) has a rather sharp edge at its lower end (see figure 6(a)); this edge gives rise to very high SE emission. In figure 4(a) this edge appears as a very bright hem around Region (1) and in figures 4(d) and (e) this hem manifests itself in sharp peaks. The superellipses of edge-on $\gamma$-pits are fitted at the maximum brightness of the bright hem

Deleted: s (1) and Deleted: meet at Deleted: ,

Deleted: which

Deleted: transition (see figures 4(a) and (c)). In-plane $\gamma$-pits will be discussed further in section 4.2.2. The superellipses are drawn around them at the outermost edge of their bright contrast (see figure 4(f)). In strongly etched specimens sometimes $\{001\}$-terraces interrupt the $\{011\}$-slopes of edge-on $\gamma$-pits (see section 4.3.1).

'[insert figures 5 and 6 about here]'

Digitising of the photographic FESEM negatives and the numerical analyses were analogous to those of the TEM images (see section 4.1), but zero was inserted for the foil-thickness $t$ and the minimum depth $w$. Since all four evaluated micrographs were taken at different areas of the same TEM-foil, they were not pasted together. The magnification was between 11,000x and 20,000x, hence each micrograph covered between about 10 and $30(\mu \mathrm{m})^{2}$ of the thin foil. It is questionable whether the four evaluated FESEM micrographs are really representative of the dispersion of $\gamma$-precipitates in the entire volume of the specimen; this concerns primarily the very few (see section 4.2.2) in-plane $\gamma$-pit images. The same four micrographs are evaluated for edge-on and for in-plane $\gamma$-pits.

4.2.1 Edge-on $\gamma$-precipitates. In total 63 edge-on $\gamma$-pits are evaluated. Due to this low number statistics are rather poor and the error limits are high. The FESEM images directly yield the chords $\sigma_{\alpha}(n, k)$, and $\sigma_{\beta}(n, k)$ defined in section 2. With the aid of equations (3b) and (3c) the half-axes $\alpha_{\mathrm{ij}}$ and $\beta_{\mathrm{ij}}$ of the $\gamma$-precipitate No. ij are calculated. With only one exception the exponents $n$ and $k$ equal 3. The averages (over micrograph No. i) $a_{\mathrm{i}}$ and $b_{\mathrm{i}}$ of the two half-axes, $R_{\mathrm{i}}$ of the aspect ratio, and $V_{\mathrm{i}}$ of the $\gamma$-volume are obtained as in section 4.1.1. 
Equations (5) are applied with $t_{\mathrm{i}}=0$ and $w=0 . w$ is assumed to vanish because even a very shallow $\gamma$-pit yields contrast. The factor 2 in equations (5c) and (5d), which stands for the two surfaces of the thin foil, cancels. The total, i.e. edge-on plus in-plane, $\gamma$-volume fraction $f_{\mathrm{i}}$ is calculated from the fraction of the area $A_{\mathrm{i}}$ of the micrograph No. i covered by edge-on $\gamma$-pit images. Equation $(5 \mathrm{~g})$ is replaced by

$$
f_{\mathrm{i}}=\frac{1.5}{A_{\mathrm{i}}} \sum_{j=1}^{N_{\mathrm{i}}} 2 \alpha_{\mathrm{ij}}^{\prime} \beta_{\mathrm{ij}}^{\prime} q_{C}(n, k) \pi
$$

The area of the evaluated edge-on $\gamma$-pits follows from equation (3e). The final averaging procedures over the four micrographs are analogous to those described in section 4.1.1, but $f_{\mathrm{i}}$ is weighted by $A_{\mathrm{i}}$ instead of by $N_{\mathrm{i}}$. The final results for the averages $a, b, R, V$, and $f$ are listed in table 1 . The quoted statistical error limits represent the standard deviations of the respective averages plus - in the case of $a$ and $b-2 \%$ for inaccuracies of the magnification of the FESEM (see section 3 ) and - in the case of $f-6 \%$ accounting for uncertainties in tracing out $A_{\mathrm{i}}$ (see section 4.1). The agreement between the SEM edge-on data and the respective TEM ones is good: $a(\mathrm{SEM})$ and $b(\mathrm{SEM})$ are $8 \%$ smaller than $a$ (TEM) and $b$ (TEM), respectively. Consequently $V(\mathrm{SEM})$ and $f(\mathrm{SEM})$ are $24 \%$ and $19 \%$, respectively, below their TEM-counterparts. $R(\mathrm{SEM})$ is very close to $R(\mathrm{TEM})$. The error limit $\sqrt{1 / 63}$ of $f(\mathrm{SEM})$ amounts to $13 \%$. With the only exception of $V($ SEM), the edge-on SEM results agree with the respective edge-on TEM ones within the statistical limits of error.

4.2.2 In-plane $\gamma$-precipitates. For reasons given above, the shape of the in-plane $\gamma$-pits is often rather poorly defined. Moreover, there are only 11 of them. Their evaluation is analogous to that of edge-on ones (see section 4.2.1). The apparent half-axes $\alpha_{\mathrm{ij}}^{\prime}$ of the imaged superellipses equal $\left[0.5 q_{\mathrm{C}}(n, n) \alpha_{\mathrm{ij}} \pi\right]$ (see equation (3a)). With one exception the exponent $n$ is 3 . No weighting procedure as in equation (5a) is performed, because it would involve the shorter half-axis $\beta_{\mathrm{ij}}^{\prime}$, which is unknown. $f_{\mathrm{i}}$ is calculated with the aid of equation (8), but the factor 1.5 is replaced by 3.0 because - in the case of in-plane $\gamma$-precipitates - the summation allows only for one of the three families of $\{001\}-\gamma$-plates. 
The overall arithmetic mean $a$ of the two long half-axes and the volume fraction $f$ are listed in table 1 . The weighting procedures and the derivations of the error limits are the same as in section 4.2.1. The result for $a$ is in excellent agreement with the respective TEM edge-on one, the error limit, however, is high. $f(\mathrm{SEM}$, in-plane) turns out to be much higher than $f$ (TEM, edge-on). The reason is that the number $N_{\text {in-plane }}=11$ of FESEM imaged in-plane $\gamma$-pits happens to be exceptionally high. The statistical average number $\left\langle N_{\text {in-plane }}>\right.$ can be estimated on the basis of $N_{\text {edge-on }}=63$ of imaged edge-on $\gamma$-pits. The ratio of the fractions of area covered by edge-on and by in-plane $\gamma$-pits is 2.0 because there are two edge-on $\{001\}$-families, but only one in-plane family. Allowing for the average aspect ratio $R_{\mathrm{ij}}=7.8$ (see table 1 ) one expects $\sigma=N_{\text {edge-on }} / N_{\text {in-plane }}=2.0 \cdot 7.8=15.6$. Evidently the present ratio $63 / 11=5.7$ happens to be far below the expected one and $f($ SEM, in-plane) is very high. The error limit $\sqrt{1 / N_{\text {in-plane }}}=\sqrt{1 / 11}$ of $f($ SEM, in-plane $)$ alone amounts to $30 \%$.

\subsection{Atomic force microscopy}

Magnetic force microscopy (MFM) will be discussed in section 4.4. Both AFMs (AFM1 and AFM2, see section 3) were operated in the tapping mode. They were alternatively equipped with both types of tips (Tip1 and Tip2, see section 3). Figure 7 is a TEM bright field image of a Tip2 before its use. Each standard individual AFM image covers $5 \mu \mathrm{m} \times 5 \mu \mathrm{m}$ of the TEM-thin foil and has $512 \times 512$ pixels. The acquisition time for the image was $10-20 \mathrm{~min}$. To get more in-plane $\gamma$-pits, micrographs were also taken at lower magnification: $15 \mu \mathrm{m} \times 15 \mu \mathrm{m}$ with $512 \times 512$ pixels each. The scan direction was chosen to be close to $<011>$, not $<001>$, because thus the shorter half-axes $\beta_{\mathrm{ij}}^{\prime}$ of the superellipses could be measured more accurately. In sections 4.3 (AFM) and 4.4 (MFM) two coordinate systems are used: $(x, y, z)$ are determined by the crystallographic axes of the specimen and $\left(x^{\prime}, y^{\prime}, z^{\prime}\right)$ are the coordinates of the AFM scan. $z^{\prime}$ equals $z$ throughout. $z^{\prime}=z=0$ characterises the average level of the $\gamma^{\prime}$-surface. In the case of AFM, $x$ ' and $y^{\prime}$ are parallel to the $[110]$ - and [110]-direction, respectively. In the case of MFM, the orientation of the $\left(x^{\prime}, y^{\prime}\right)$-axes relative to the crystallographic axes varies from specimen to specimen (see section 4.4). A topography AFM image is presented in figure $8(a)$. The difference in contrast between edge-on and in-plane $\gamma$-pits is due to the fact that those areas which lie more than $52 \mathrm{~nm}$ below the $\gamma^{\prime}$-surface are shown in white and only 
edge-on $\gamma$-pits reach to this depth. After subtraction of the background, neighbouring original micrographs were 'pasted together' in the computer (see section 4.1). Four TEM-foils were studied at both magnifications.

'[insert figures 7, 8, and 9 about here]'

Figure 9 shows an edge-on $\gamma$-pit imaged with AFM2 equipped with a supersharp Tip1. The magnification was high: $512 \times 512$ pixels covered $1 \mu \mathrm{m} \times 1 \mu \mathrm{m}$ of the TEM-thin foil. The lines of equal height clearly indicate the three regions defined in section 4.2: Region (1) - flat bottom, Region (2) - ascent / descent approximately parallel to \{011\}, Region (3) $\gamma^{\prime}$-surface. The transition between Region (1) and Region (2) is very well defined.

Basically the numerical evaluation procedures are analogous to those applied to the FESEM images (see section 4.2). However, the derivation of the apparent half-axes $\alpha_{\mathrm{ij}}^{\prime}$ and $\beta_{\mathrm{ij}}^{\prime}$ from the three-dimensional numerical AFM output data $z^{\prime}\left(x^{\prime}, y^{\prime}\right)$ requires detailed analyses of the three issues (i)-(iii) listed below. Whereas the contrast evaluated for TEM and FESEM images stems mainly from the lower parts of the etched $\gamma$-pits, the AFM yields information on their upper parts. This will be discussed below. It depends on the surface profile of the specimen which point of the tip is closest to the specimen during the AFM scan. This point may be in the outermost sphere-shaped end of the tip or in its pyramidical shaft. At the point of closest approach the slopes $\partial z^{\prime} / \partial x^{\prime}$ of the tip and of the specimen surface are the same $[11,30] ;$, here the scan is supposed to be along the $x^{\prime}$-axis. The AFM computer registers the nominal coordinates $\left(x^{\prime}, y^{\prime}, z^{\prime}\right)$. If the tip were infinitely slender and not inclined (see section 3$),\left(x^{\prime}, y^{\prime}, z^{\prime}\right)$ would represent the profile of the specimen exactly. The function $z^{\prime}\left(x^{\prime}\right)$ actually registered in a linear scan depends on (i) the original shape of the $\gamma$-precipitate, (ii) the amount of $\gamma^{\prime}$-matrix which dissolved next to the $\gamma$-precipitate, and (iii) the shape and inclination of the tip. Issues (i) and (ii) determine the shape of the $\gamma$-pit.

'[insert figure 10 about here]'

4.3.1 Edge-on $\gamma$-precipitates. Some AFM profiles $z(x, \operatorname{sim})$ were computer simulated for edge-on $\gamma$-pits and Tip2 shown in figure 7. It is inclined by $10^{\circ}$ relative to the normal of the 
specimen; this agrees with the actual geometry of AFM2. The full width of the $\gamma$-pit equals the present average full smaller axis $2 b=52.2 \mathrm{~nm}$ (see table 1 ). In the simulations the scan direction is parallel to the $x$-(not $x^{\prime}$-!)direction. In figure 10(a) the $\gamma$-pit/ $\gamma^{\prime}$-matrix interfaces are exactly normal to the $x$-direction. The profile in figure 10(b) is similar, but the top edges of the $\gamma$-pit are blunted. Their slopes have the indices $\{011\}$. Such blunting is brought about by a partial dissolution of the $\gamma^{\prime}$-matrix because atoms at sharp edges are less strongly bound (see section 4.1). The micrographs presented in figures 2, 4, 5, 8, and 9 clearly show such blunting, mainly in the direction of the smaller half-axis $\beta_{\mathrm{ij}}$. Along the longer half-axis

$\alpha_{\mathrm{ij}}$ blunting is less pronounced, moreover $\alpha_{\mathrm{ij}}$ is close to $8 \beta_{\mathrm{ij}}$ (see table 1). Hence blunting causes hardly any problem in the determination of $\alpha_{\mathrm{ij}}$. Figure 10 shows the profiles $z(x, \operatorname{sim})$. Due to the finite opening angle (half-cone angle: $\approx 12^{\circ}$ ) and the inclination of the tip, the maximum depth is $69 \mathrm{~nm}$ and $124 \mathrm{~nm}$ in figures 10 (a) and (b), respectively. Because of the inclination of the tip the slopes of the right and left flanks of $z(x, \operatorname{sim})$ are not the same.

If a foil had been very strongly etched, the slopes of the blunted edges do not have the indices $\{011\}$, but $\{111\}$, i.e. the most densely packed planes are exposed. $\{111\}$-planes intersect the (001)-surface of the specimen along <011>-directions. This may give the outline of edge-on $\gamma$-pits a zigzag appearance and in-plane ones become approximately circular (see $\gamma$-pit C in figure 8(a)) or change their orientation (see $\gamma$-pit D in figure 8(a)).

In contrast to the computer simulated AFM-profiles $z(x$, sim) shown in figure 10, observations by AFM revealed that in Region (2) (see figure 6 and section 4.2) the ascents and descents of strongly etched specimens are terraced: the $\{011\}$-planes are interrupted by horizontal (001)-planes (see figure 9(c)). The (001)-terrace in figure 9(c) extends over about 5 pixels. This result could be obtained neither by TEM nor by SEM. Due to these (001)-terraces the overall angles between the ascents, respectively descents, and the $\gamma$-surface are often less than $45^{\circ}$. Based on the results of the computer simulations and on analytical considerations the following procedures for obtaining the apparent half-axes $\alpha_{\mathrm{ij}}^{\prime}$ and $\beta_{\mathrm{ij}}^{\prime}$ and the exponents $n$ and $k$ are adopted.

Determination of $\beta_{\mathrm{ij}}^{\prime}$ : About seven (in the following this number will be referred to by the letter $v, v \approx 7$ ) edge-on $\gamma$-pits of various sizes are chosen randomly in each original 
$5 \mu \mathrm{m} \times 5 \mu \mathrm{m}$ micrograph and the profiles $z(x)$, respectively $z(y)$ (see figure (8b)), along the shorter axes are calculated from the $z^{\prime}\left(x^{\prime}, y^{\prime}\right)$-AFM data. For each of the $v \approx 7 \gamma$-pits, the $z$-level $\zeta_{\mathrm{m}}<0,{ }_{-} 1 \leq m \leq 2 v$, is marked at which the slope $|\partial z / \partial x|$, respectively $|\partial z / \partial y|$, changes markedly. This change indicates the sharp transition from Region (1) to Region (2) sketched in figure 6(a) and defined in section 4.2. $\zeta_{0}$ is the average over $\zeta_{\mathrm{m}}, 1 \leq m \leq 2 v$. The factor 2 before $v$ is due to the fact that each $\gamma$-pit has two slopes: ascent and descent. At the level $z=\zeta_{0}, \beta_{\mathrm{ij}}^{\prime}$ is measured for all edge-on $\gamma$-pits in the considered $5 \mu \mathrm{m} \times 5 \mu \mathrm{m}$ micrograph. The standard deviation of $\zeta_{0}$ within one original $5 \mu \mathrm{m} \times 5 \mu \mathrm{m}$ micrograph amounts to about $5 \%$. Due to differences in etching, the scatter of $\zeta_{0}$ found for different TEM-foils is larger: $\zeta_{0}$ ranges from $-40 \mathrm{~nm}$ to $-100 \mathrm{~nm}$. It is reiterated that etching was not intentional, but a mere side effect of the standard twin-jet electro-polishing procedure of TEM-foils. If etching had been geared to AFM, $\left|\zeta_{0}\right|$ would have been small and reproducible (see section 5). For $z<\zeta_{0}$, the slopes $\partial z / \partial x$ and $\partial z / \partial y$ are determined by the half-cone angle of the tip and its inclination. Those parts of the $\gamma$-pits which lie below the level $\zeta_{0}=-52 \mathrm{~nm}$, are white in figure $8(a)$.

Determination of $\alpha_{\mathrm{ij}}^{\prime}$ : As stated above, $\alpha_{\mathrm{ij}}^{\prime}$ can easily be measured. $\alpha_{\mathrm{ij}}^{\prime}$ is visually derived from those points in the micrographs (see figures $8(a)$ and (b)) where the contrast changes rapidly; this is analogous to the measurement of $\beta_{\mathrm{ij}}^{\prime}$, but there is no need to establish the profiles $z(x)$ and $z(y)$. The level $|z|$ at which $\alpha_{\mathrm{ij}}^{\prime}$ is measured, is about one half of $\left|\zeta_{0}\right|$ used for $\beta_{\mathrm{ij}}^{\prime}$.

Exponents $n$ and $k$ : Since $\alpha_{\mathrm{ij}}^{\prime}$ and $\beta_{\mathrm{ij}}^{\prime}$ are determined at different levels $z, n$ and $k$ cannot be obtained by AFM. Based on the TEM results (see section 4.1.1), throughout the evaluations 3 is inserted for $n$ and $k$.

Ten pasted together micrographs taken for four thin TEM-foils are evaluated; they contain 1829 edge-on $\gamma$-pits. The calculations are the same as described in section 4.2.1 for edge-on $\gamma$-pits imaged by FESEM. The only differences concern the calculations of the statistical limits of error: that of the magnification has been given in section 3 and that of the area $A_{\mathrm{i}}$ appearing in equation (8) is $4.5 \%$.The final results are listed in table 1 . The agreement 
of the AFM results with the respective TEM ones is good. The average half-axes $a$ and $b$ are $11 \%$ below and $6 \%$ above the respective TEM data. The $\gamma$-volume fraction $f$ (AFM, edge-on) is $17 \%$ smaller than $f$ (TEM, edge-on). The average $\gamma$-precipitate volume $V$ (AFM, edge-on) agrees within its statistical error limit with $V$ (TEM, edge-on). The average aspects ratio $R$ (AFM, edge-on) is $14 \%$ below $R$ (TEM, edge-on); this difference reflects those concerning the half-axes $a$ and $b$. There is no systematic dependence of $a, b$, or $f$ on the AFM or on the tip used. The distribution functions $\Phi\left(\alpha_{\mathrm{ij}} / a\right)$ and $\Phi\left(\beta_{\mathrm{ij}} / b\right)$ are shown in figure 11. For comparison with the distributions determined by TEM for edge-on $\gamma$-precipitates (see figure 3), log-normal distribution functions are plotted in figure 11 with the same parameters as in figure 3. Evidently there is very good agreement between the distributions measured by AFM and TEM.

[insert figure 11 about here]

About every other pasted together area had to be disregarded because $\zeta_{0}$ could not be accurately determined. About the same fraction of thin foils proved to be unsatisfactory for TEM work. Moreover, it must be kept in mind that one searches each foil already in the TEM for electron transparent areas and disregards thick ones. In the AFM investigation, however, areas with unsatisfactory $\gamma$-pits are only recognised during their evaluation. If the preparation of the present $\mathrm{Ni}_{69} \mathrm{Co}_{9} \mathrm{Al}_{18} \mathrm{Ti}_{4}$-specimens had been geared to the AFM instead of to the TEM the fraction of good AFM images would have been higher.

4.3.2 In-plane $\gamma$-precipitates. Three types of in-plane $\gamma$-pits are distinguished in figure $8(a)$ :

(i) at $\mathrm{B}$, (ii) at $\mathrm{C}$, and (iii) at $\mathrm{D}$.

(i) B: This $\gamma$-pit is similar to that marked $\mathrm{K}$ in figure 2, but that at $\mathrm{B}$ has been etched more strongly.

(ii) C: This $\gamma$-pit is rather shallow and approximately circular. Its ascents and descents have strong $\{111\}$-components. $\{111\}$-planes intersect the surface of the specimen along $<011>$-directions. The apparent longer half-axis $\alpha_{\mathrm{ij}}^{\prime}$ is measured along $<001>$-directions.

(iii) D: Regions (2) (see section 4.2 and figure 6(b)) of this $\gamma$-pit are parallel to 
$\{111\}$-planes. This $\gamma$-pit is very deep. $\alpha_{\mathrm{ij}}^{\prime}$ is measured along the $<001>$-diagonals.

Twenty-eight $15 \mu \mathrm{m} \times 15 \mu \mathrm{m}$ AFM-images are evaluated, they are not pasted together. The micrographs were taken for four different TEM-foils. A total of 402 in-plane $\gamma$-pits is evaluated. The half-axes $\alpha_{\mathrm{ij}}^{\prime}$ are measured as described in section 4.3.1. The further numerical evaluations are the same as in section 4.2.2. The results for the average longer half-axis $a$ (AFM, in-plane) and for the $\gamma$-volume fraction $f$ (AFM, in-plane) are listed in table 1 . The error limit of the area $A_{\mathrm{i}}$ (see equation (8)) is 5\%. The results agree satisfactorily with the respective edge-on TEM ones: $a(\mathrm{AFM}$, in-plane) is $15 \%$ too high and $f(\mathrm{AFM}$, in-plane) is $7 \%$ too low.

[insert figure 12 about here]

\subsection{Magnetic force microscopy}

As stated in section 3, AFM2 was equipped with a tip which had been coated with a 40nm thick hard magnetic cobalt alloy layer. As in section 4.3 , the coordinate system $\left(x^{\prime}, y^{\prime}, z^{\prime}\right)$ is that of the AFM scan and $(x, y, z)$ is determined by the crystallographic axes of the specimen; $z$ ' equals $z$. Since the specimen had only been polished, but not etched (see section 3), it could not be orientated relative to the scanning direction by using the optical microscope attached to AFM2. Hence there is no general correlation between $(x, y)$ and $\left(x^{\prime}, y^{\prime}\right)$. The tip and the specimen were magnetised with a small permanent magnet such that different poles meet during the measurements. Each line was scanned twice: first the profile $z^{\prime}\left(x^{\prime}, y^{\prime}=\right.$ const. $)$ was registered in the standard tapping mode and subsequently the tip was raised to the level $z_{0}$ and scanned at the constant level $z_{0}$, i.e. during the second scan the tip did not contact the specimen. The magnetic interaction of the tip with the specimen alters the resonant frequency of the cantilever and thus leads to a phase shift $\varphi$ between it and the driving frequency. During the second scan, $\varphi\left(x^{\prime}, y^{\prime}=\right.$ const. $)$ was registered. In figure 12(a) a standard tapping mode topography $z^{\prime}\left(x^{\prime}, y^{\prime}\right)$-image and in figures $12(\mathrm{~b}),(\mathrm{d})$, and (f) $\varphi\left(x^{\prime}, y^{\prime}\right)$-images are shown; $\varphi$ varies between $+0.1^{\circ}$ and $-0.15^{\circ}$. Since neither details of the magnetic properties of the $\gamma$ - and $\gamma^{\prime}$-phase nor of the tip are known, $\varphi$ could not be calculated as a function of the shape and the size of the $\gamma$-precipitates. The same two areas $A_{1}$ and $A_{2}$ of one specimen were 
evaluated for edge-on and in-plane $\gamma$-pits; $A_{1}$ consisted out of one and $A_{2}$ out of nine pasted together $5 \mu \mathrm{m} \times 5 \mu \mathrm{m}$ micrographs. The error limit of $A_{\mathrm{i}}$ is $4.5 \%$.

4.4.1 Edge-on $\boldsymbol{\gamma}$-precipitates. In total there are 131 of them. The superellipses are drawn such that they include the whole area in which $\varphi$ is strongly negative, i.e. below some limit $\omega_{0} \approx-0.02^{0}$ (see figure $\left.12(\mathrm{~d})\right)$. $\omega_{0}$ varies slightly with $z_{0}$. On the basis of the TEM results (see section 4.1.1) the exponents $n$ and $k$ are set equal to 3 . A superellipse is outlined in figure 12(d). The further evaluations follow those applied to the FESEM images of edge-on $\gamma$-pits (see section 4.2.1). The results have been compiled in table 1 . That for the longer half-axis $a(\mathrm{MFM})$ is in excellent agreement with that obtained by TEM, but the result for the shorter half-axis $b$ (MFM) exceeds $b$ (TEM) by $48 \%$. Consequently the average aspect ratio

$R(\mathrm{MFM})$ is too low and the average volume $\left.V_{\alpha} \mathrm{MFM}\right)$ too high. The volume fraction $f(\mathrm{MFM})$ in turn agrees perfectly with $f(\mathrm{TEM})$. But since $b(\mathrm{MFM})$ is much too high, the latter agreement must be considered as fortuitous. The statistical error limit of $f(\mathrm{MFM})$ amounts to $42 \%$.

In spite of the low number of edge-on $\gamma$-precipitates studied by MFM and the uncertainties concerning the interpretation of the $\varphi$-contrast, the result for $a$ is surprisingly accurate. The strong deviation of $b(\mathrm{MFM})$ from the three other data listed in table 1 for $b$ of edge-on $\gamma$-pits, indicates systematic short comings of the present MFM evaluations. Since the main components of the magnetic stray fields produced by edge-on $\gamma$-precipitates lie probably in planes normal to them, the evaluation of $a$ is expected to be far less critical than that of $b$. Moreover, the smaller size of $b$ makes it more sensitive to all types of errors.

4.4.2 In-plane $\gamma$-precipitates. There are only 20 of them. The superellipses are drawn at the level $\varphi \approx-0.02^{\circ}$ (see section 4.4.1). An example is outlined in figure 12(f). The further numerical evaluations are analogous to those performed for FESEM images (see section 4.2.2). The in-plane MFM results for the average longer half-axis $a$ and for the $\gamma$-volume fraction $f$ have been compiled in table 1. $a$ and $f$ exceed the respective edge-on TEM results by $11 \%$ and by $66 \%$. Evidently the agreement concerning $a$ is good. Hence the large discrepancy concerning $f$ must be due to errors in the number of evaluated in-plane $\gamma$-pits. On the basis of the TEM investigations the ratio $\sigma$ of the numbers of edge-on to in-plane $\gamma$-pits is expected to be 15.6 (see section 4.2.2), which equals 2.4 times the present ratio 
$\sigma=131 / 20=6.6$ (see section 4.4.1 and above). A possible reason why there were so many in-plane $\gamma$-pits is that even those of them which lay entirely below the surface of the specimen may have produced magnetic stray fields which were registered by the MFM.

\section{Discussion}

The microscopic characterisation of second phase particles involves two steps:

(i) generation of some contrast and (ii) relating it to the size and shape of the particles. In most cases step (ii) is quite complex. One of the few exceptions are $\mathrm{L}_{2}$-long-range ordered $\gamma$-precipitates embedded in the disordered $\gamma$-matrix of nickel based superalloys (see section 4.1): by TEM these $\gamma^{\prime}$-precipitates are dark-field imaged with one of their superlattice reflections. The difficulties encountered in measuring the $\gamma^{\prime}$-volume fraction $f_{\gamma}$, by AFM have been demonstrated by Hazotte et al. [8], who determined $f_{\gamma}$, of cuboidal $\gamma^{\prime}$-precipitates in an industrial nickel based superalloy. Their edge-length was about 500nm. The experimental results for $f_{\gamma}$, were found to decrease as the depth of etching increased. The correct value for $f_{\gamma}$, was obtained by extrapolating this depth to zero. Even in the relatively easy case of dark-field TEM of spherical $\gamma^{\prime}$-precipitates with average radii above $5 \mathrm{~nm}$, the error limit of $f_{\gamma^{\prime}}$, is at best around $20 \%$ [2].

For the time being magnetic force microscopy is left aside; it will be discussed towards the end of this section. The present plate-shaped $\gamma$-precipitates in $\mathrm{Ni}_{69} \mathrm{Co}_{9} \mathrm{Al}_{18} \mathrm{Ti}_{4}$ have nearly the same chemical composition as the $\gamma^{\prime}$-matrix and both phases have the f.c.c. crystal structure. Hence in order to image the $\gamma$-precipitates by SEM and AFM, surface profiles had to be generated. In the TEM these profiles yield mass thickness contrast and the $\gamma$-parts remaining embedded in the $\gamma^{\prime}$-matrix give rise to some strain contrast. The present surface profiles were created unintentionally by etching during the standard electro-polish of thin TEM-foils. Since the same specimens were meant to be investigated by TEM, SEM, and AFM, their preparation had to be geared to TEM. During electro-polishing the $\gamma$-precipitates dissolved faster than the $\gamma$ '-matrix; hence $\gamma$-pits appeared (see section 3). The habit planes are of the type $\{001\}$. The upper parts of the edge-on $\gamma$-pits are blunted: they are bounded by $\{011\}$-planes or - in the case of strongly etched specimens - by $\{111\}$-planes (see sections 4.2 and 4.3). The contrast produced by the $\gamma$-pits was successfully related to the size and 
shape of the $\gamma$-precipitates (see section 4). The procedures are different for TEM, SEM, and AFM and for edge-on and in-plane $\gamma$-pits.

First edge-on $\gamma$-pits are discussed. The present description of the plate-shaped $\gamma$-precipitates as superellipsoids turned out to be very convenient. All four relevant parameters, i.e. the two average half-axes $a$ and $b$ and the two average exponents $n$ and $k$ (equation (1)), can be derived from micrographs of edge-on $\gamma$-pits alone [25] They also yield Deleted: 4 the $\gamma$-volume fraction. For reasons given in section 4.3.1, $n$ and $k$ were presently not determined by AFM. The contrast observed by TEM and SEM stems mainly from the deeper parts of the $\gamma$-pits, whereas the AFM 'sees' only their surface-near parts. Therefore specimens meant to be studied by AFM should be etched very lightly. In this case blunting of the $\gamma$-pits is slight; hence the parameter $\zeta_{0}$ introduced in section 4.3 .1 is close to zero and the exponents $n$ and $k$ can be determined by AFM.

The results obtained by TEM for edge-on $\gamma$-pits are considered as the most reliable ones. The corresponding SEM and AFM results for the half-axes $a$ and $b$ and the $\gamma$-volume fraction $f$ agree well with the respective TEM data (see table 1 and sections 4.2 and 4.3). This proves that AFM is well suited for the characterisation of nano-scale plate-shaped second phase particles. No systematic short comings of this technique became evident. For spherical particles analogous results were reported earlier [11,12].

Since the present preparation schedule had been geared to TEM, the AFM images had to be evaluated as detailed in section 4.3.1. If, however, standard metallographic polishing and etching procedures are carried out, the evaluations of AFM images can be automated. This is a great advantage, especially in routine work. In contrast to TEM and SEM, AFM images can be taken almost automatically. This compensates the advantage of the TEM, which images many $\gamma$-pits and $\gamma$-precipitates simultaneously. Because of the abrupt changes in the surface profile at the $\gamma$-pits, it is unlikely that the scan rates can be raised significantly while a high resolution is kept. All procedures described in section 4 can also be applied to particles which have the shape of cubes with rounded edges and corners. The only difference is that the two half-axes $\alpha$ and $\beta$ are the same. The $\gamma^{6}$-precipitates in advanced nickel based superalloys are such rounded cubes [2,7-9]. 


\section{Conclusions}

1. The size, shape, and volume fraction of nano-scale plate-shaped second phase particles can be accurately measured by atomic force microscopy. The achieved accuracy is 
comparable to that of transmission electron microscopy. In general slightly etching is required.

2. If the magnetic properties of the nano-scale plate-shaped particles differ sufficiently from those of the matrix, they can also be characterised by magnetic force microscopy. In this case no etching is needed.

3. Nano-scale particles of any convex shape, e.g. $\gamma$ '-cubes with rounded edges and corners in nickel based superalloys, can be elegantly described as superellipsoids [25]. A superellipsoid is characterised by four independent parameters, all of which can be measured in just one micrograph. With the aid of equations (1) - (3) all relevant geometric parameters of such particles can easily be calculated.

ACKNOWLEDGEMENTS. The authors thank Mrs. A. Ricker (Institut für Medizinische Physik und Biophysik ) for technical assistance in the AFM work, Mrs. L. Rettich (Institut für Materialphysik), and Mrs. G. Kiefermann (Institut für Medizinische Physik und Biophysik) for photographic work. Financial support by the Deutsche Forschungsgemeinschaft and by the research program MSM 0021620834 of the Ministry of Education of the Czech Republic is gratefully acknowledged. 
REFERENCES

[1] A.J. Ardell, Metall. Trans. A 162131 (1985).

[2] E. Nembach, Particle Strengthening of Metals and Alloys (Wiley, New York, 1997).

[3] J.L. Walter, M.R. Jackson and C.T. Sims (Editors), Alloying (ASM INTERNATIONAL, Metals Park, 1988).

[4] A.G. Khachaturyan, Theory of Structural Transformations in Solids (Wiley, New York, 1983).

[ 5] D.M. Barnett, J.K. Lee, H.I. Aaronson and K.C. Russel, Scripta Met. 81447 (1974).

[ 6] J.K. Lee, D.M. Barnett and H.I. Aaronson, Met. Trans. 8A 963 (1977).

[ 7] M. Göken and H. Vehoff, Scripta Mater. 35983 (1996).

[ 8] A. Hazotte, M. Troyon and A. Bourhettar, Micros. Microanal. Microstruct. 7377 (1996).

[ 9] M. Göken and M. Kempf, Acta Mater. 471043 (1999).

[10] K. Durst and M. Göken, Prakt. Metallogr. 38197 (2001).

[11] U. Lagerpusch, B. Anczykowski and E. Nembach, Phil. Mag. A 812613 (2001).

[12] B. Fruhstorfer, V. Mohles, R. Reichelt and E. Nembach, Phil. Mag. A 822575 (2002).

[13] J. Wosik, H.J. Penkalla, K. Szot, B. Dubiel, F. Schubert and A. CzyrskaFilemonowicz, Prakt. Metallogr. 39140 (2002).

[14] W. Liu, H. Rösner and E. Nembach, Z. Metallkde. 88648 (1997).

[15] T. Pretorius, D. Baither and E. Nembach, Acta Mater. 491981 (2001).

[16] D. Baither, T. Pretorius and E. Nembach, Acta Mater. 511229 (2003).

[17] F.R.N. Nabarro and M.S. Duesbery (Editors), Dislocations in Solids, Vol. 10 (Elsevier, Amsterdam, 1996).

[18] R. Wagner and R. Kampmann, in Materials Science and Technology, Vol. 5, R.W. Cahn, P. Haasen and E.J. Kramer (Editors), (VCH Verlagsgesellschaft, Weinheim, 1991), p. 213.

[19] M. Fährmann, W. Hermann, E. Fährmann, A. Boegli, T.M. Pollock and H.G. Sockel, Mater. Sci. Eng. A 260212 (1999).

[20] D. Siebörger, H. Knake and U. Glatzel, Mater. Sci. Eng. A 29826 (2001).

[21] P. Hein, Dansk Kunsthandvaerk 3797 (1964).

[22] L. Rosendahl, Appl. Math. Modelling 2411 (2000). 
(Kluwer, Dordrecth, 2000), p. 13.

[24] T. Wriedt, Part. Part. Syst. Charact. 19256 (2002)

[25] I. Sobchenko, J. Pesicka, D. Baither, R. Reichelt and E. Nembach, Appl. Phys. Letters, 89133107 (2006)

[26] E.W. Weisstein, Superellipsoid (MathWorld _A Wolfram Web Resource, 2006); http://mathworld.wolfram.com/Superellipsoid.html.

[27] C.C. Jia, K. Ishida and T. Nishizawa, Metall. Mat. Trans. A 25473 (1994).

[28] M. Doi, Prog. Mater. Sci. 40 79 (1996).

[29] L. Reimer, Scanning Electron Microscopy, second edition (Springer, Berlin, 1998).

[30] D. Keller, Surface Sci. 253353 (1991).

\section{Deleted: 3}

Deleted: 4

Deleted: in press

Deleted: 5

Deleted: 6

Deleted: 7

Deleted: 8

Deleted: 29 
Table 1: Results for the half-axes $a$ and $b$, the aspect ratio $R$, the volume $V$, and the volume fraction $f$ ( total, i.e. edge-on plus in-plane ) of the $\gamma$-precipitates. Statistical limits of error are quoted.

\begin{tabular}{|c|c|c|c|c|c|c|c|c|}
\hline & \multicolumn{2}{|c|}{ TEM } & \multicolumn{2}{|c|}{ SEM } & \multicolumn{2}{|c|}{ AFM } & \multicolumn{2}{|c|}{ MFM } \\
\hline & edge-on & in-plane & edge-on & in-plane & edge-on & in-plane & edge-on & in-plane \\
\hline$a[\mathrm{~nm}]$ & $209 \pm 6$ & $220 \pm 6$ & $192 \pm 19$ & $208 \pm 40$ & $185 \pm 12$ & $240 \pm 17$ & $211 \pm 25$ & $232 \pm 17$ \\
\hline$b[\mathrm{~nm}]$ & $26.1 \pm 0.7$ &.$\%$ & $24.1 \pm 1.8$ &.$\%$ & $27.7 \pm 2.7$ &.$\%$ & $39 \pm 3$ &.$\%$ \\
\hline$R$ & $7.8 \pm 0.1$ &.$\%$ & $7.8 \pm 0.7$ &.. & $6.7 \pm 0.3$ &.$\%$ & $5.4 \pm 0.3$ &.$\%$ \\
\hline$V\left[10^{6} \mathrm{~nm}^{3}\right]$ & $9.6 \pm 0.7$ &.$\%$ & $7.3 \pm 1.5$ &.$\%$ & $8.3 \pm 2.0$ &.$\%$ & $12.6 \pm 2.8$ &.$\%$ \\
\hline$f$ & $0.035 \pm 0.008$ &.$\%$ & $0.029 \pm 0.008$ & $0.075 \pm 0.036$ & $0.030 \pm 0.004$ & $0.033 \pm .004$ & $0.036 \pm 0.015$ & $0.059 \pm 0.016$ \\
\hline
\end{tabular}




\section{FIGURE CAPTIONS}

Fig. 1: Superellipsoids, the ratio $\alpha / \beta$ of the half-axes equals 5.0. Cross sections through the centre: (a) edge-on, (b) in-plane view. (c) Three-dimensional view. The exponents $n$ and $k$ are defined in equation (1); in (a) the parameter $q_{\mathrm{V}}(n, k)$ (equation (2a)) is given below each sketch.

Fig. 2: Bright field TEM image of plate-shaped $\gamma$-precipitates and $\gamma$-pits in $\mathrm{Ni}_{69} \mathrm{Co}_{9} \mathrm{Al}_{18} \mathrm{Ti}_{4}$. The Miller indices of the plane of the thin foil are (001) and the electron beam is normal to it. The $x$ - and $y$-axes are parallel to <001>-directions. Thickness of the foil: $352 \mathrm{~nm}$. For A - K see section 4.1. For one edge-on (L) $\gamma$-pit and one in-plane (H) $\gamma$-precipitate the superellipses are outlined; the magnification of these two small micrographs is 2.1times that of the large one.

Fig. 3: Distribution functions (a) $\Phi\left(\alpha_{\mathrm{ij}} / a\right)$ and (b) $\Phi\left(\beta_{\mathrm{ij}} / b\right)$ of edge-on $\gamma$-precipitates measured by TEM. The dashed lines represent log-normal distributions functions fitted to the data. (c) $\beta_{\mathrm{ij}}$ is plotted versus $\alpha_{\mathrm{ij}}$, each point represents one $\gamma$-precipitate, $\diamond$ indicates the overall averages $a$ and $b$ and the curve shows equation (6). Less than about $0.5 \%$ of the data lie outside the ranges of the diagrams.

Fig. 4: High resolution FESEM secondary electron images of (a) edge-on and (b) in-plane $\gamma$-pits in $\mathrm{Ni}_{69} \mathrm{Co}_{9} \mathrm{Al}_{18} \mathrm{Ti}_{4}$. The $x$ - and $y$-axes are parallel to $<001>$-directions. (c) The superellipse for $\gamma$-pit A is outlined, (d) intensity $I(x)$ of the brightness across A, (e) $I(y)$ across A, (f) superellipse for B, (g) $I(x)$ across B. $I$ is in arbitrary units.

Fig. 5: Red-green anaglyph of an FESEM stereo pair of edge-on $\gamma$-pits. The stereo tilt angle is $\pm 5^{\circ}$.

Fig. 6: Schematic sketch of the surface profile at (a) an edge-on and (b) an in-plane $\gamma$-pit. The numbers indicate those of the 'Regions' defined in section 4.2.

Fig. 7: TEM bright field image of an AFM Tip2 before its use. 
Fig. 8: (a) AFM (AFM2 with Tip2) topography image of $\gamma$-pits in $\mathrm{Ni}_{69} \mathrm{Co}_{9} \mathrm{Al}_{18} \mathrm{Ti}_{4}$. The $x$ - and $y$-axes are parallel to $<001>$-directions. Areas which lie deeper than $z=-52 \mathrm{~nm}$, are shown in white. The $\gamma^{\prime}$-surface is at the level $\mathrm{z}=0$. A marks an edge-on $\gamma$-pit and B-D in-plane ones. (b) Outline of the superellipse of the edge-on $\gamma$-pit A and its profile $z(y)$, (c) outline of the superellipse of the in-plane $\gamma$-pit $\mathrm{C}$ and its profile $z(x)$.

Fig. 9: High magnification AFM (AFM2 with Tip1) topography image of an edge-on $\gamma$-pit. Lines of equal height are indicated; the difference in height between neighbouring lines is $15 \mathrm{~nm}$. Areas which lie deeper than $z=-110 \mathrm{~nm}$, are shown in black. The $\gamma^{\prime}$-surface is at the level $\mathrm{z}=0$. The arrow in (c) points at a (001)-terrace.

Fig. 10: Computer simulated surface profiles. The actual geometry of Tip2 (see figure 7) with $10^{\circ}$ inclination is used. Actual surface profile $z(x)$, - - - - simulated AFM-profile $z(x, \operatorname{sim})$.

Fig. 11: Distribution functions (a) $\Phi\left(\alpha_{\mathrm{ij}} / a\right)$ and (b) $\Phi\left(\beta_{\mathrm{ij}} / b\right)$ of edge-on $\gamma$-precipitates measured by AFM. The dashed lines represent the log-normal distribution functions fitted to the TEM data (see figure 3).

Fig. 12: (a,b) Images of a just polished $\mathrm{Ni}_{69} \mathrm{Co}_{9} \mathrm{Al}_{18} \mathrm{Ti}_{4}$-specimen taken with AFM2 equipped with a magnetic tip: (a) tapping mode, (b) $\varphi$-shift mode, the $x$ - and $y$-axes are parallel to $<001>$-directions. (c) Height-profile $z^{\prime}\left(x^{\prime}\right)$ through (a). (d) Outline of the superellipse of the edge-on $\gamma$-pit A in (b), (e) its profile $\varphi(x)$, (f) and (g) as (d) and (e), respectively, for the in-plane $\gamma$-pit B in (b). Areas with $\varphi<-0.1^{\circ}$ are black in (b) and (d). 
(a)

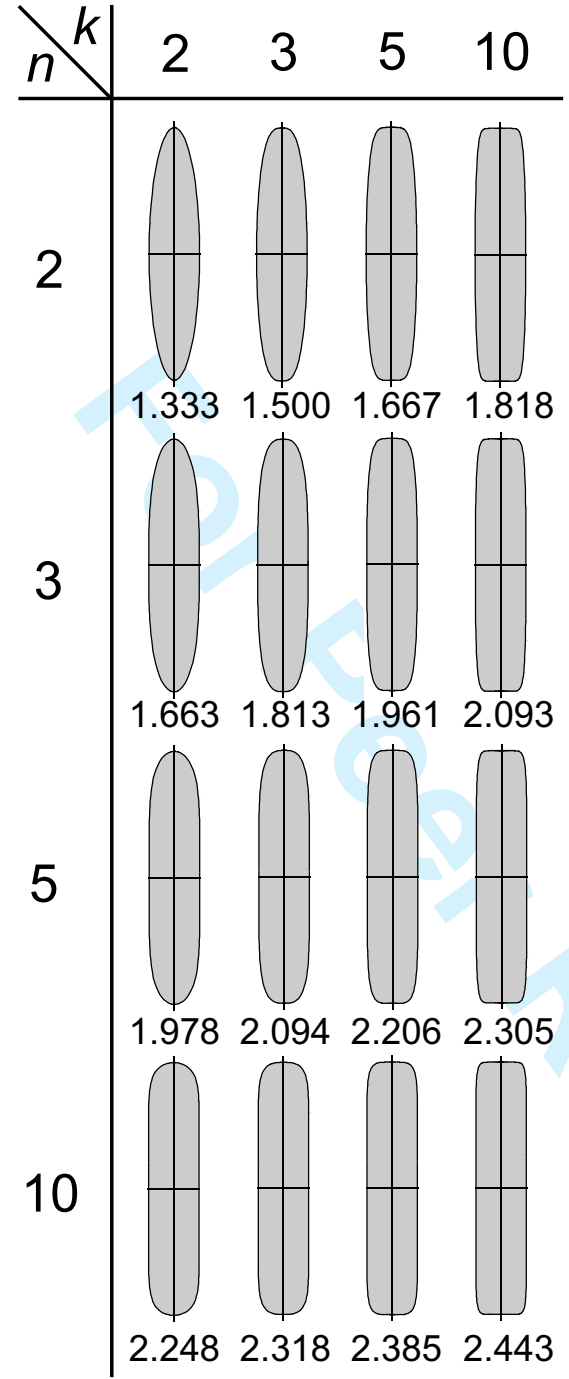

(c)

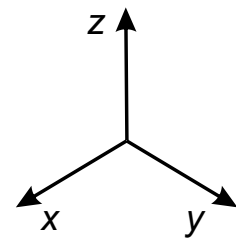

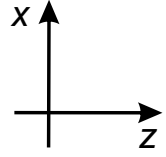

(b)

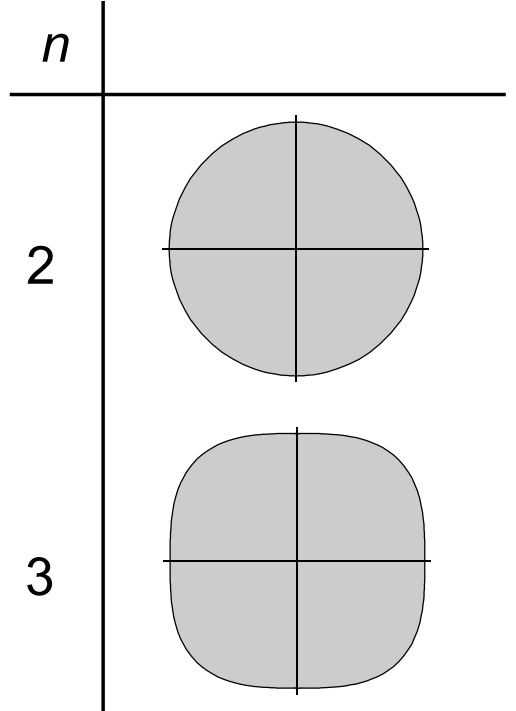

5

10
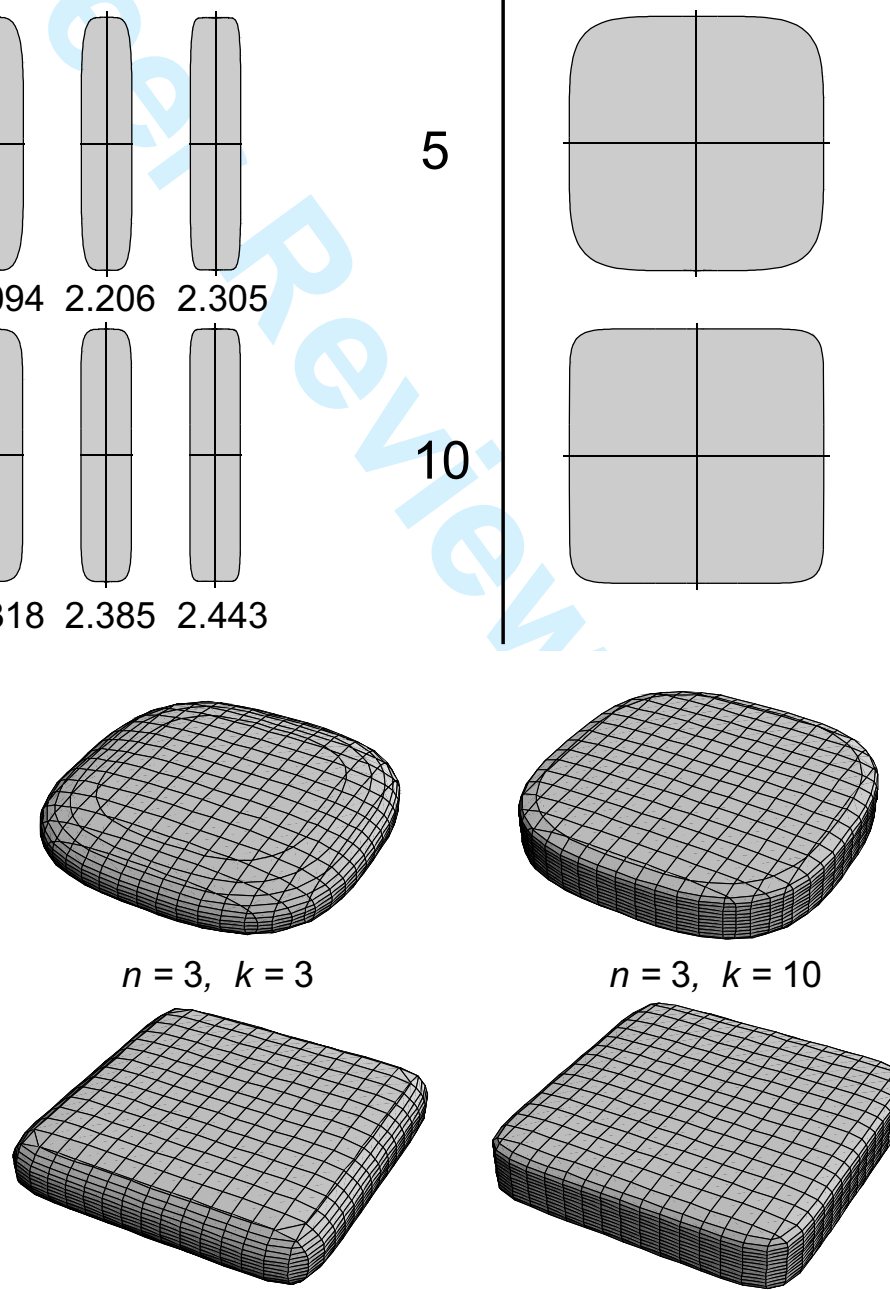

$n=10, k=3$

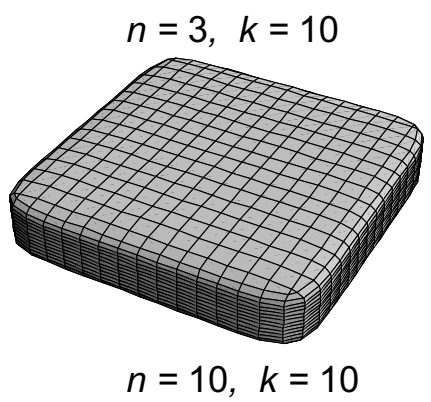

http://mc.manuscriptcentral.com/pm-pml 


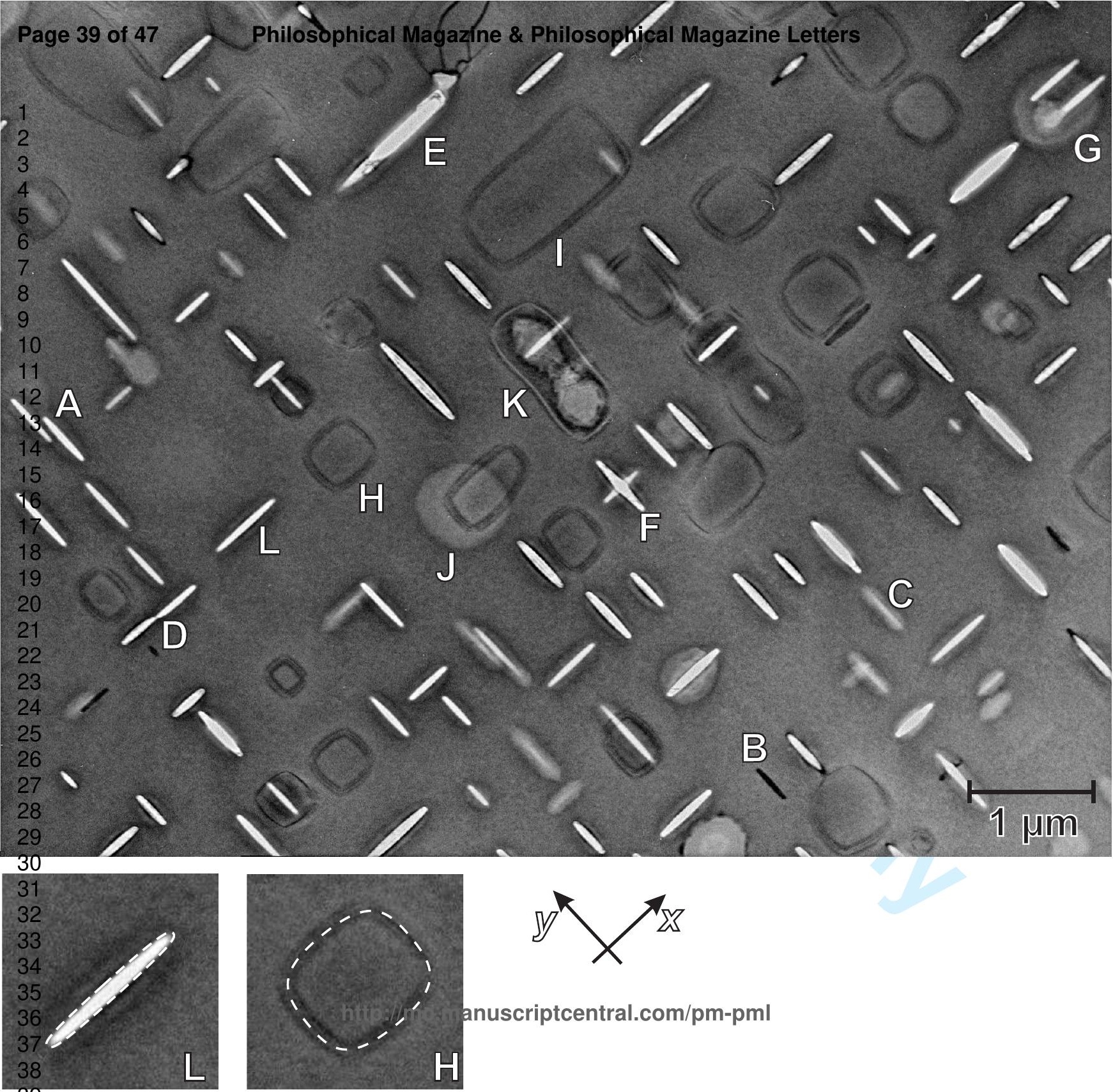




\section{hilosophical Magazine \& Philosophical Nragmzinedfelfer}

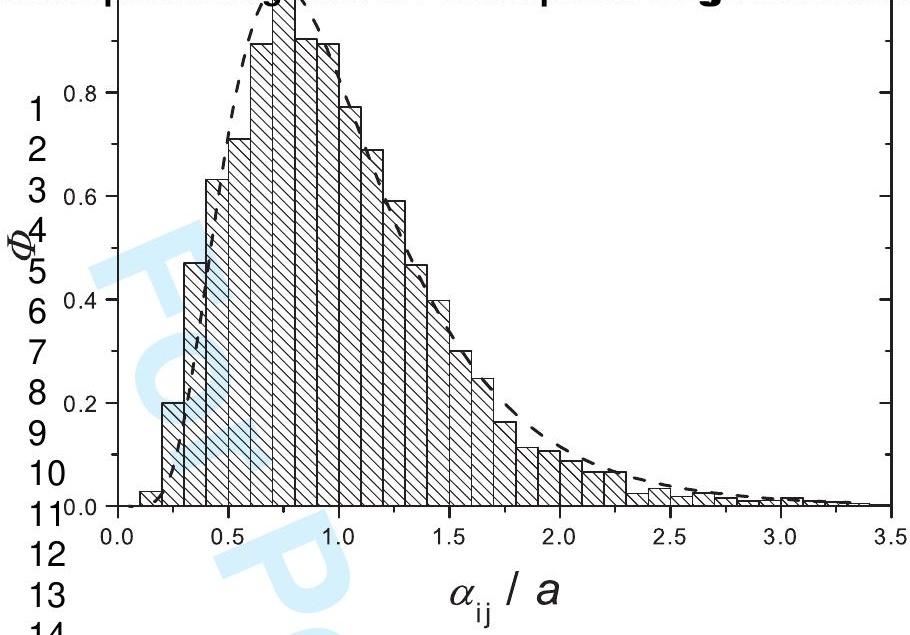

14

15

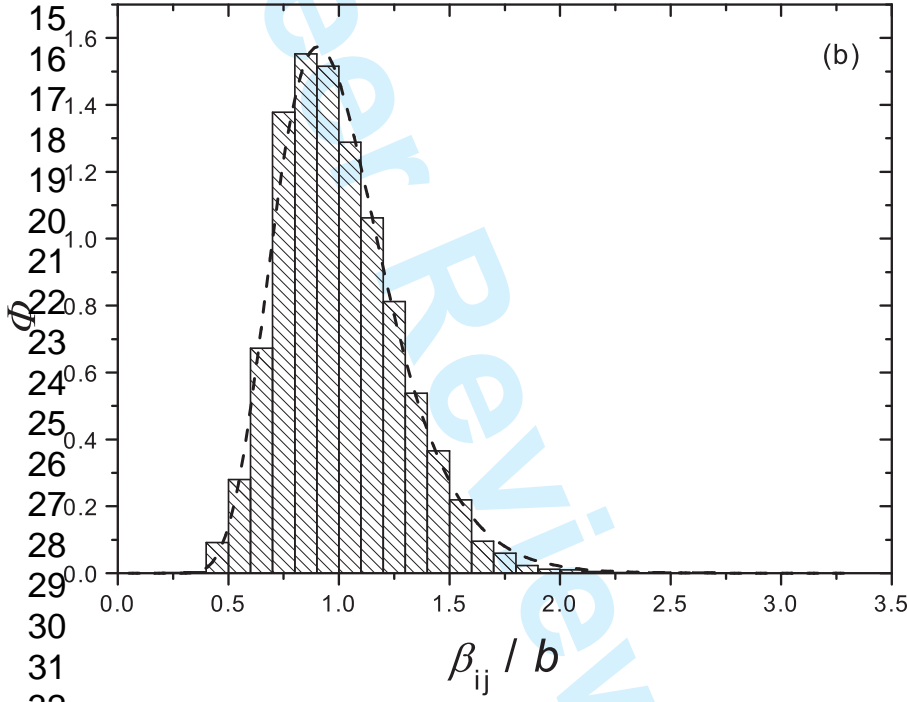

32

3

3

3

3

3

3 i

8

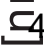



(4)

43

44

45

46

4

4

49

(c)

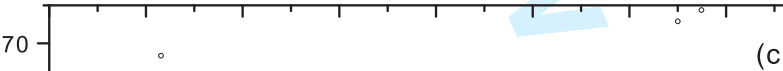

0 http://ma,manyscriptceptral_com/pm-pm

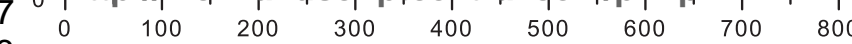




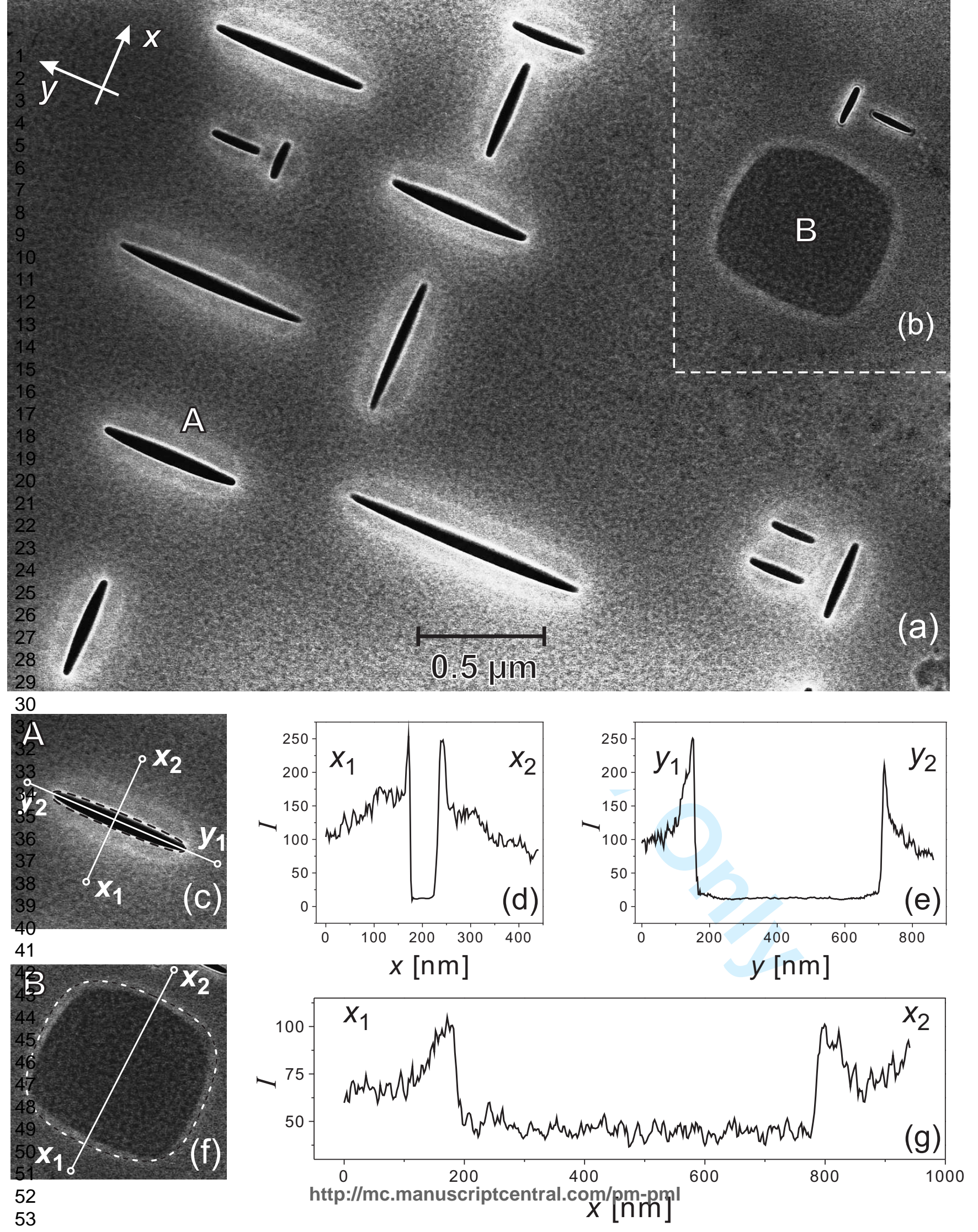




\section{Philosophical Magazine \& Philosophical MagPeajigedRedfełs}

1
2
3
4
5
6
7
8
9

10

11

12

13

14

15

16

17

18

19

20

21

22

23

24

25

26

2

28 


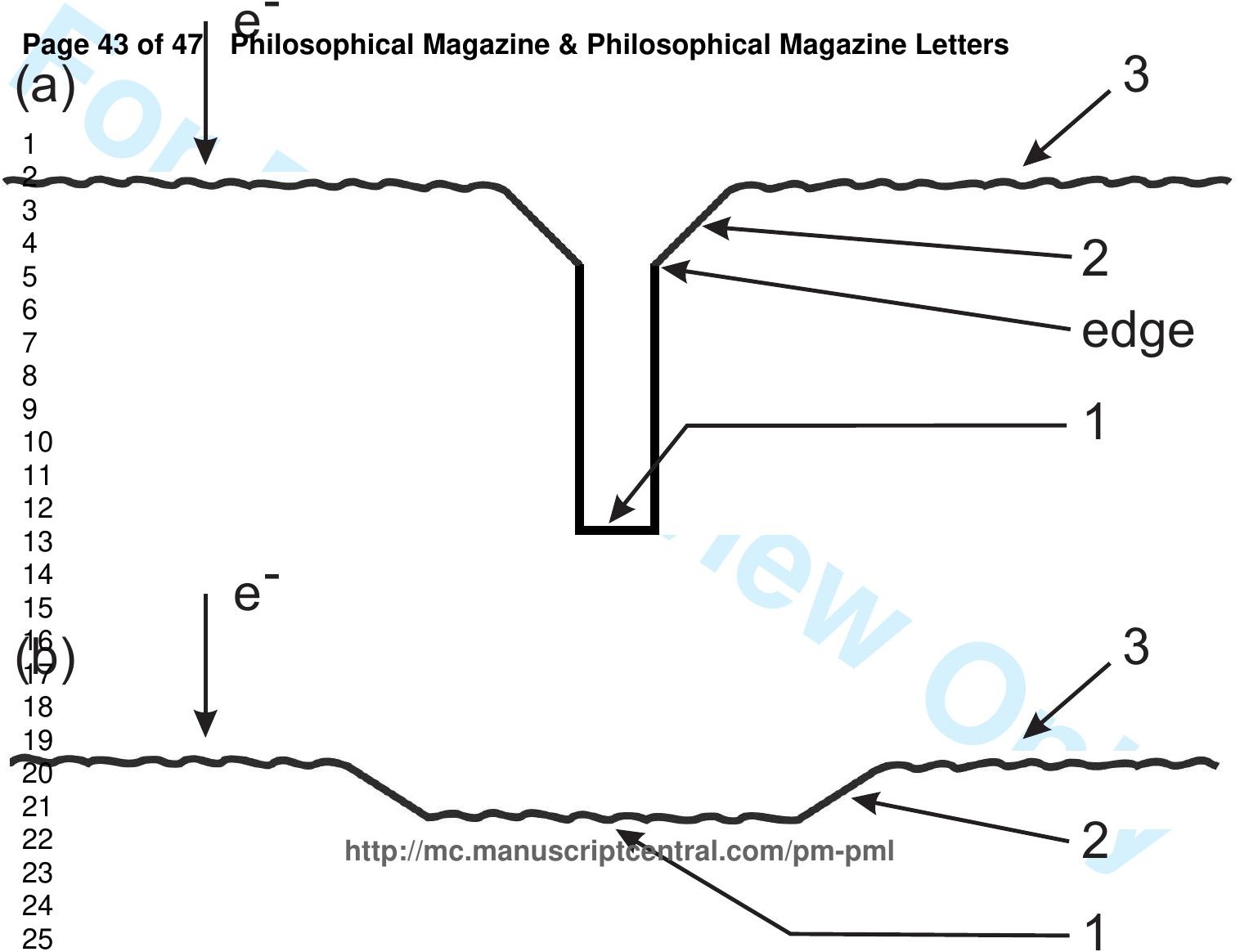


21

22

23

24

25 26 2 


\section{Page 45 of 47 Éfilosophical Magazine \& Philosophical Magazine Letters}

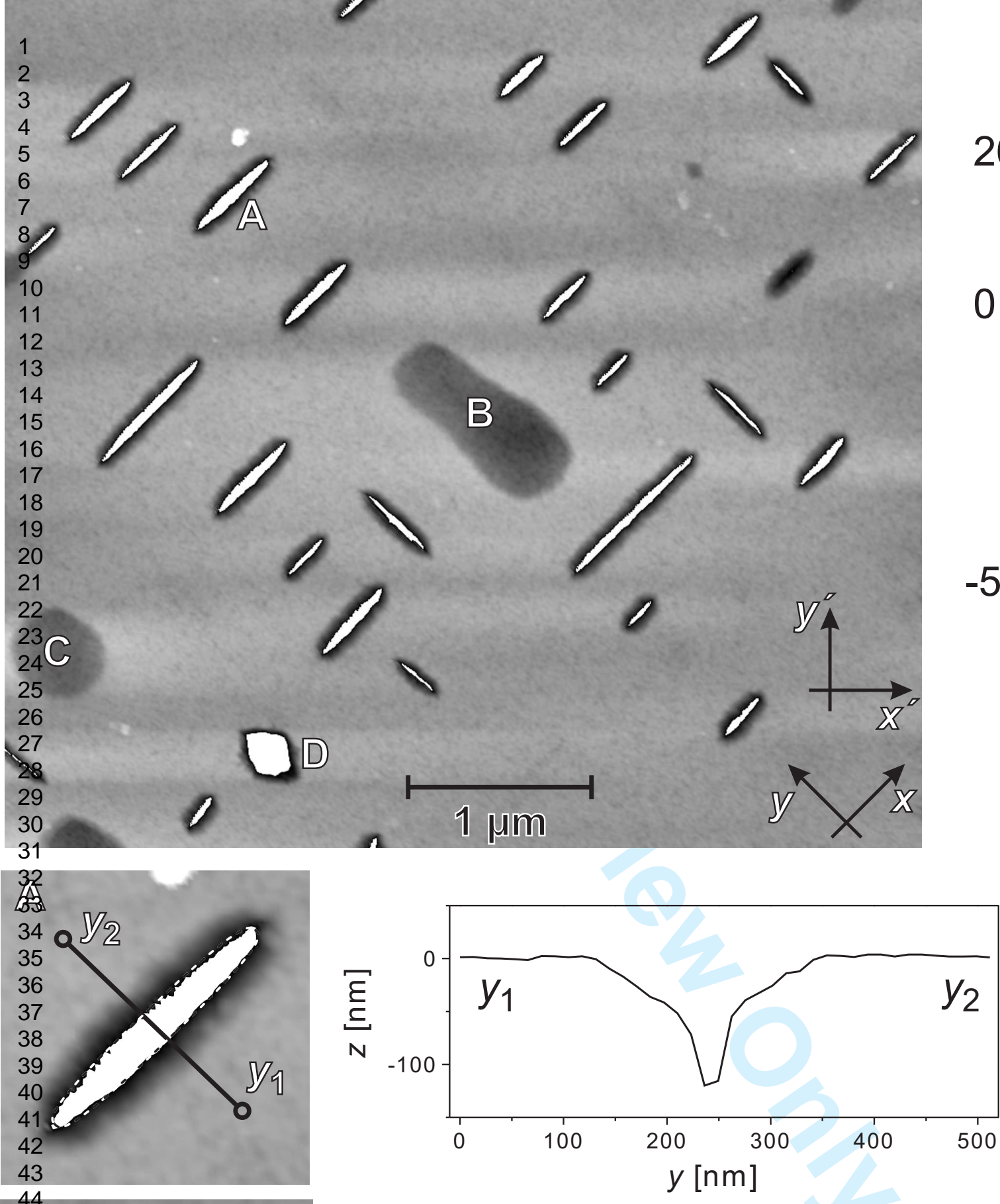

(a)

46

47

48

49

'50

51

52

53

54

5

56

57

58

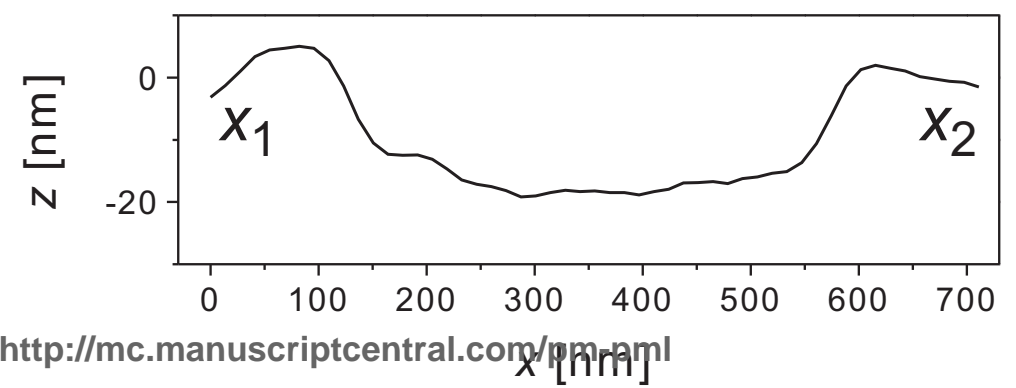

(b)

(c) 
Philosophical Magazine \& Philosophical Magazine Letages 46 (6૯4) 7

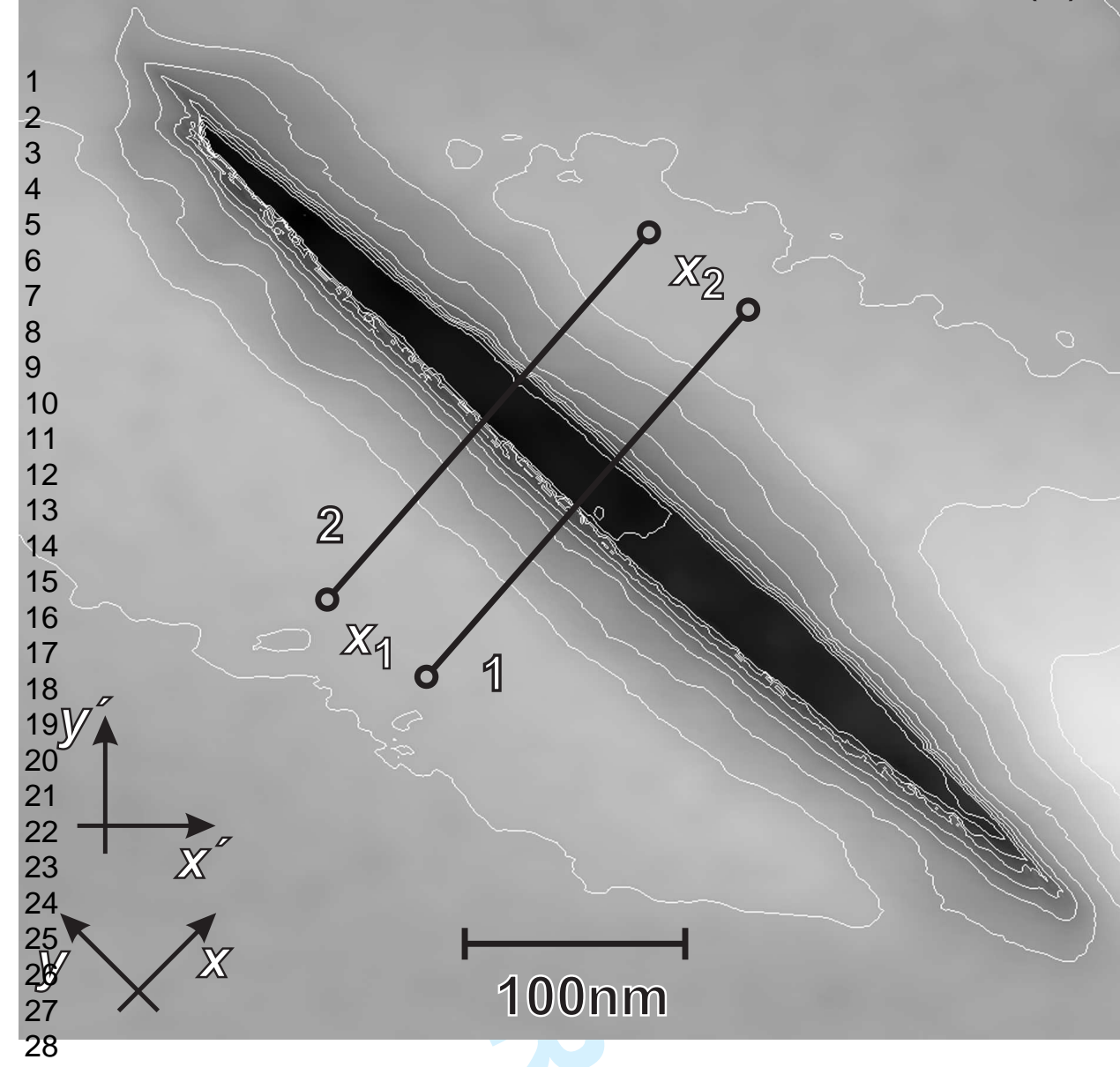

29

3

3

32

33

34

3

35
36

3
3

3

38

39

40

41

42

43

44

45

46

47

48

49

5

518

52 ᄃ

$53 \mathrm{~N}$

54

55

56

57

58

59

60
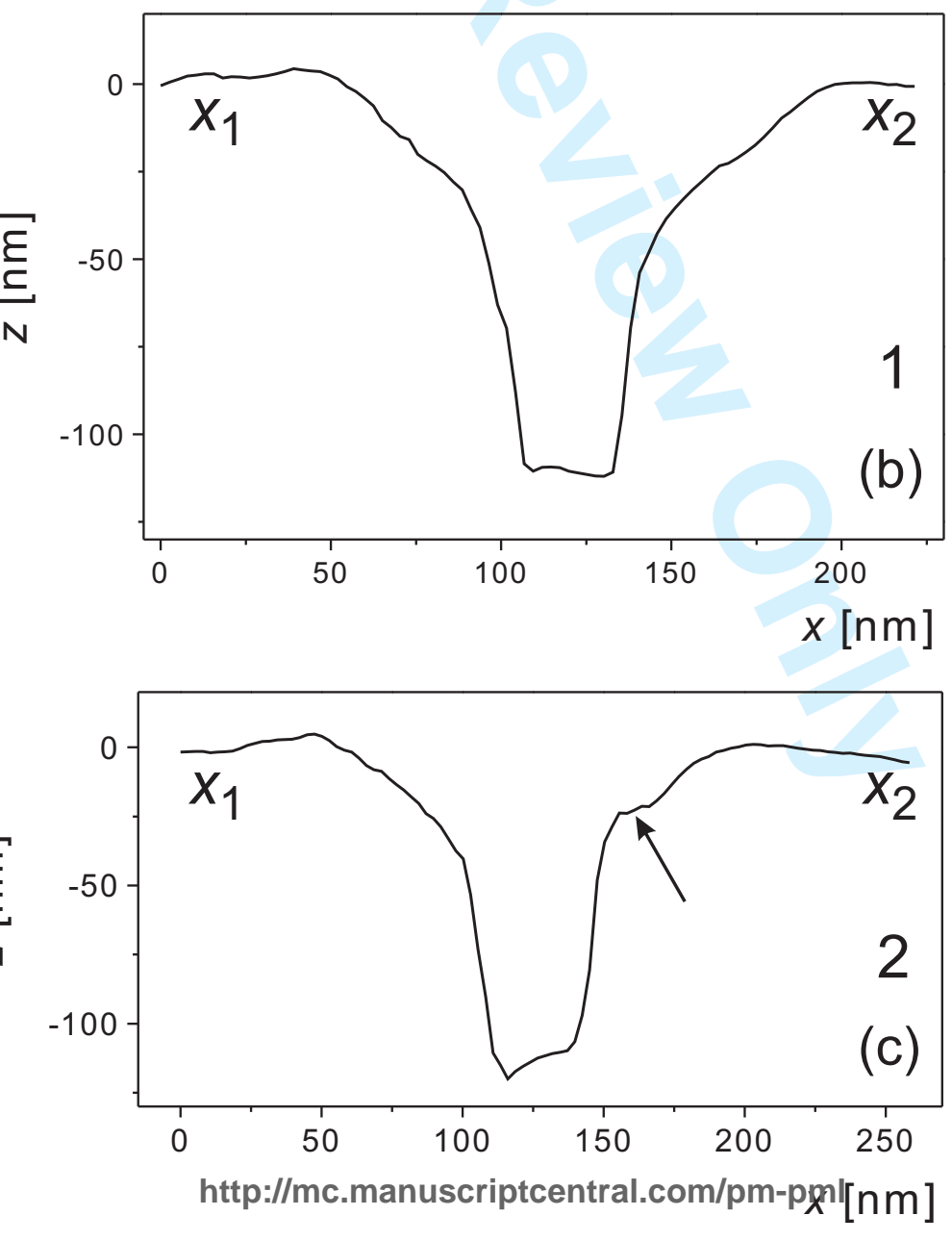
Page 47 of 47

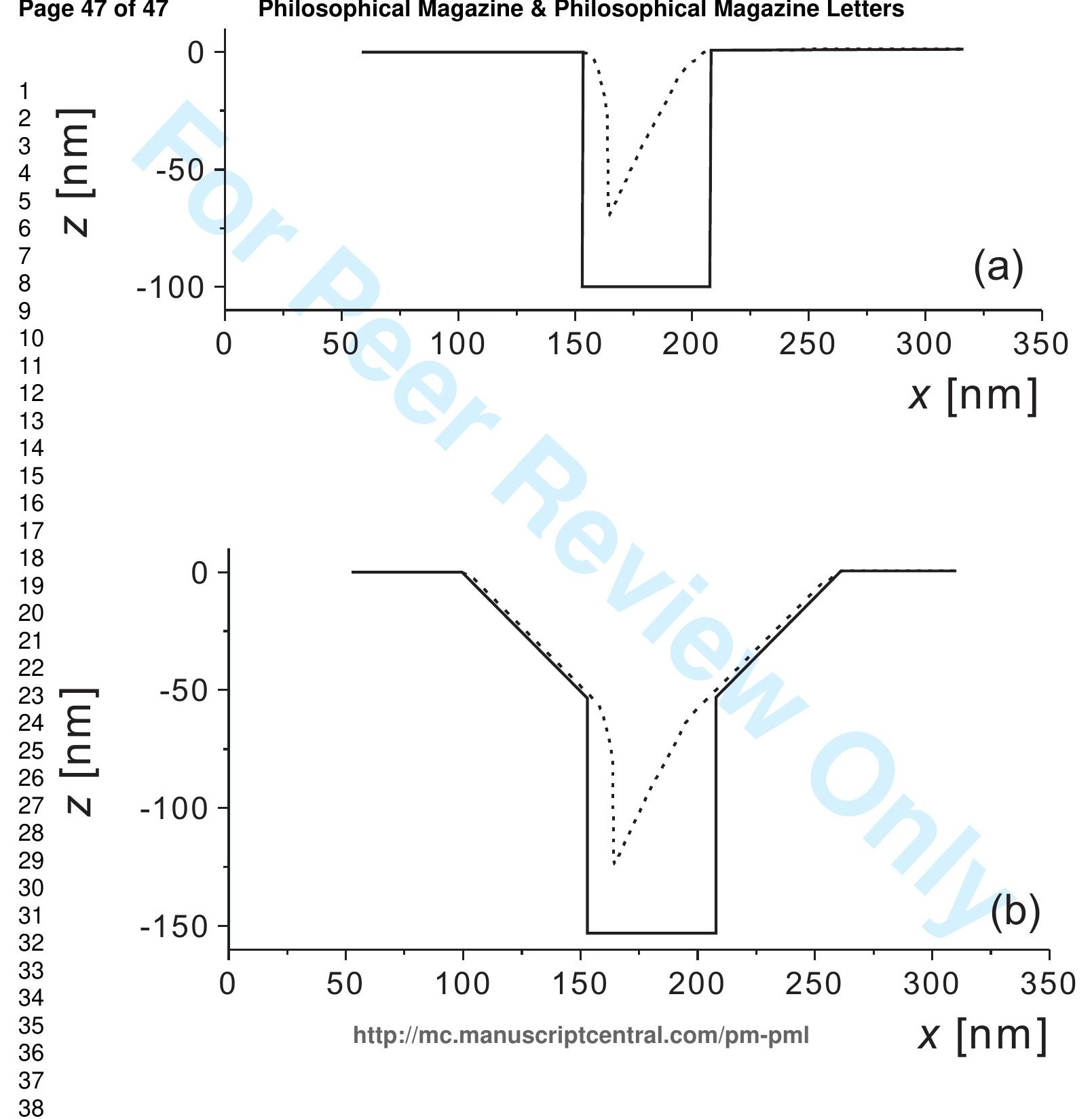


hilosophical Magazine \& Philosophical Ragez46eotzattel

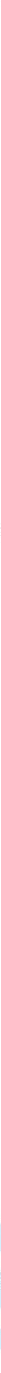

